ARTICLE

https://doi.org/10.1038/s41467-019-09713-w

\title{
A DNMT3A PWWP mutation leads to methylation of bivalent chromatin and growth retardation in mice
}

Gintarè Sendžikaitė (1) ${ }^{1}$, Courtney W. Hanna (i) ${ }^{1,2}$, Kathleen R. Stewart-Morgan (1) 1,3, Elena Ivanova ${ }^{1}$ \& Gavin Kelsey (10) 1,2

DNA methyltransferases (DNMTs) deposit DNA methylation, which regulates gene expression and is essential for mammalian development. Histone post-translational modifications modulate the recruitment and activity of DNMTs. The PWWP domains of DNMT3A and DNMT3B are posited to interact with histone 3 lysine 36 trimethylation (H3K36me3); however, the functionality of this interaction for DNMT3A remains untested in vivo. Here we present a mouse model carrying a D329A point mutation in the DNMT3A PWWP domain. The mutation causes dominant postnatal growth retardation. At the molecular level, it results in progressive DNA hypermethylation across domains marked by H3K27me3 and bivalent chromatin, and de-repression of developmental regulatory genes in adult hypothalamus. Evaluation of non- $\mathrm{CpG}$ methylation, a marker of de novo methylation, further demonstrates the altered recruitment and activity of DNMT3AD329A at bivalent domains. This work provides key molecular insights into the function of the DNMT3APWWP domain and role of DNMT3A in regulating postnatal growth.

\footnotetext{
${ }^{1}$ Epigenetics Programme, Babraham Institute, Cambridge CB22 3AT, UK. ${ }^{2}$ Centre for Trophoblast Research, University of Cambridge, Cambridge CB2 3EG, UK. ${ }^{3}$ Present address: Biotech Research \& Innovation Centre, 2200 Copenhagen, Denmark. Correspondence and requests for materials should be addressed to G.K. (email: gavin.kelsey@babraham.ac.uk)
} 
$\mathrm{D}$ NA methylation is an epigenetic modification that can regulate gene expression by inhibiting transcription factor binding and mediating changes of chromatin conformation during development ${ }^{1,2}$. The inactive $\mathrm{X}$ chromosome, imprinted genes, transposons, and repetitive elements are silenced by DNA methylation, together with additional epigenetic modifications. Furthermore, DNA methylation plays a key role in regulating tissue-specific gene expression during development, allowing differential cellular fate determination during lineage specification ${ }^{3-6}$. Methylation is added de novo to DNA at carbon5 of cytosine bases by DNA methyltransferase (DNMT) 3A, DNMT3B and DNMT3C, although the last is confined to the murine male germline $e^{7-10}$. DNA methylation is subsequently maintained by DNMT1, which localises at the replication fork, recognises hemi-methylated DNA and methylates the nascent strand $^{11-14}$. The last member of the DNMT protein family is DNMT3L, a catalytically inactive but essential co-factor for gametic methylation events ${ }^{15-17}$. DNMT3A and DNMT3B show highly conserved structural similarity and partial functional redundancy, but there are biological contexts in which one protein predominates ${ }^{18-20}$. DNMT3B is the major DNMT required for DNA methylation programming during embryogenesis and Dnmt3b $3 b^{-/-}$mice die prior to embryonic day 11.5 (E11.5) ${ }^{18,20}$. DNMT3A is predominantly responsible for DNA methylation in oogenesis ${ }^{21,22}$ and neurogenesis ${ }^{23}$, and $D n m t 3 a^{-1-}$ mice fail to survive more than a few weeks after birth ${ }^{18}$. Mutations in human DNMT3A are uniquely associated with haematological malignancies and growth abnormalities ${ }^{24-27}$, while DNMT3B mutations cause the Immunodeficiency, Centromeric instability, Facial anomalies (ICF) syndrome ${ }^{28}$.

DNMT3A and DNMT3B can methylate cytosines in both CpG and $\mathrm{CpH}$ contexts, but show rather limited specificity over flanking sequence composition ${ }^{29-31}$. Thus, post-translational modifications (PTMs) of histone tails are believed to regulate the recruitment of DNMT3s through interactions with their ADD (ATRX-DNMT3-DNMT3L) and PWWP (Pro-Trp-Trp-Pro motif) domains ${ }^{32}$. The ADD domain contains a motif homologous to plant-homeodomain (PHD) zinc fingers, and is shared between DNMT3s and the alpha thalassaemia/mental retardation syndrome X-linked (ATRX) protein ${ }^{33,34}$. The ADD domain of all DNMT3s positively interacts with the amino-terminal tail of histone 3 but is inhibited by histone 3 lysine 4 (H3K4) dimethylation or tri-methylation, marks typically associated with active chromatin ${ }^{34-37}$. The catalytic domain of DNMT3A is autoinhibited by the ADD domain and upon the recognition of unmethylated $\mathrm{H} 3 \mathrm{~K} 4$ a conformation change unmasks the catalytic site ${ }^{37,38}$

The PWWP domain is found in DNMT3A, DNMT3B and many histone methyl-transferase or acetyl-transferase proteins ${ }^{39}$. In DNMT3A and DNMT3B, the domain is required for their localisation to major satellite repeats at pericentromeres ${ }^{40,41}$. A mutation in the PWWP domain of DNMT3B was identified in a familial ICF syndrome case, where patients showed loss of DNA methylation at satellite repeat regions ${ }^{42}$. In general, PWWP domains have been shown to interact with methylated histone tails, including but not limited to H3K36me3, a mark found along actively transcribed genes $39,43,44$. Biochemical studies, including chromatin precipitation and peptide arrays, have reproducibly demonstrated that the PWWP domain of DNMT3A interacts exclusively with H3K36me2/34-47. Furthermore, a point mutation (D329A) in a conserved aspartic acid residue in the PWWP domain abrogated the ability of the DNMT3A-PWWP domain to bind H3K36me2 and $\mathrm{H} 3 \mathrm{~K} 36 \mathrm{me} 3$ peptides or pull down $\mathrm{H} 3 \mathrm{~K} 36 \mathrm{me} 2 / 3$-containing native nucleosomes ${ }^{44-47}$. The D329A mutation also reduced the preferential heterochromatin localisation of DNMT3A2 in transfected cells and decreased catalytic activity on unmethylated native nucleosomes ${ }^{44}$. Surprisingly, it was subsequently shown that when assayed in mouse embryonic stem (ES) cells, the PWWP domain of DNMT3B, but not of DNMT3A, was responsible for H3K36me3 recognition and associated gene body methylation, a mechanism that was also successfully modelled in yeast ${ }^{48,49}$. However, whilst mutations in the DNMT3B-PWWP domain or H3K36me3 depletion resulted in loss of gene body methylation in ES cells and yeast $^{48,49}$, in epiblast-like stem cells de novo methylation was reported to occur over transcribed regions in the absence of H3K36me $3^{50}$. Thus, the role of the PWWP domain in targeting de novo DNMTs to chromatin remains enigmatic and, in particular, highlights the necessity for in vivo studies to investigate the mechanisms targeting DNMT3A to chromatin and the function of the DNMT3A PWWP domain.

In this study, we generate mice carrying a point mutation $\left(D n m t 3 a^{D 329 A}\right)$ in the DNMT3A-PWWP domain, which unexpectedly is gain of function and manifests phenotypically as dominant postnatal growth retardation. We show that DNMT3A $\mathrm{D}^{\mathrm{D} 29 \mathrm{~A}}$ targets $\mathrm{H} 3 \mathrm{~K} 27 \mathrm{me} 3$ and bivalent chromatinmarked domains for DNA hypermethylation, which is associated with loss of repression of developmental transcription factor genes and altered histone PTM landscape. Thus, a mutation in DNMT3A predicted to abrogate an interaction with H3K36me2/3 causes a complex, gain-of-function phenotype in vivo associated with recruitment of DNMT3A towards H3K27me3-marked chromatin.

\section{Results}

Generation and phenotypic analysis of Dnmt $3 a^{D 329 A}$ mice. To investigate the in vivo significance of the DNMT3A PWWP domain, we generated a mouse carrying the previously in vitro characterised D329A mutation ${ }^{44-47}$ in the endogenous Dnmt3a locus (Fig. 1a and Supplementary Fig. 1). Mating Dnmt3a $a^{+/ D 329 A}$ heterozygous males with wild-type females resulted in 19 out of 23 confirmed pregnancies producing a litter, with an average litter size of 8 . In contrast, only 2 out of 19 confirmed Dnmt $3 a^{+/ D 329 A}$ female pregnancies produced a litter, with an average litter size of 2 (Supplementary Table 1). Paternal transmission litters showed over $50 \%$ post-weaning survival, whilst there were no survivors on maternal transmission. Dnmt $3 a^{+/ D 329 A}$ mothers consistently went overdue and experienced labour dystocia. To assess whether a problem in parturition accounted for the maternal transmission deficit, we performed caesarean section on Dnmt $3 a^{+/ D 329 A}$ females and transferred offspring to foster mothers. This resulted in $45 \%$ post-natal litter survival, suggesting that the parturition failure is a dominant effect in females carrying the D329A mutation (Supplementary Table 2), a phenotype that is not explored further in this study.

We proceeded by transmitting the D329A mutation from males crossed to females with heterozygous oocyte-specific conditional ablation of Dnmt3a $(\Delta)^{21,22}$, thus generating four genotypes amongst offspring: $+/+, \Delta /+,+/ D 329 A$ and $\Delta /$ $D 329 A$ (where the maternal allele is listed first); the last carries the D329A mutation as the only functional Dnmt3a allele (Fig. 1b). Notably, Dnmt $3 a^{+/ D 329 A}$ and Dnmt $3 a^{\Delta / D 329 A}$ mice displayed significant postnatal growth retardation, which was more severe in the Dnmt3a $a^{\Delta / D 329 A}$ genotype and particularly pronounced in males (Fig. 1c). In contrast, Dnmt $3 a^{\Delta /+}$ offspring were indistinguishable from Dnmt $3 a^{+/+}$, indicating no haploinsufficiency of Dnmt3a at a phenotypic level, and showing that the $D 329 A$ allele exhibited a gain of function. We confirmed that the growth deficit of Dnmt3a+/D329A and Dnmt $3 a^{\Delta / D 329 A}$ mice was not associated with reduced serum growth hormone or insulin-like growth factor 1, major modulators of postnatal growth (Supplementary Fig. 2). 

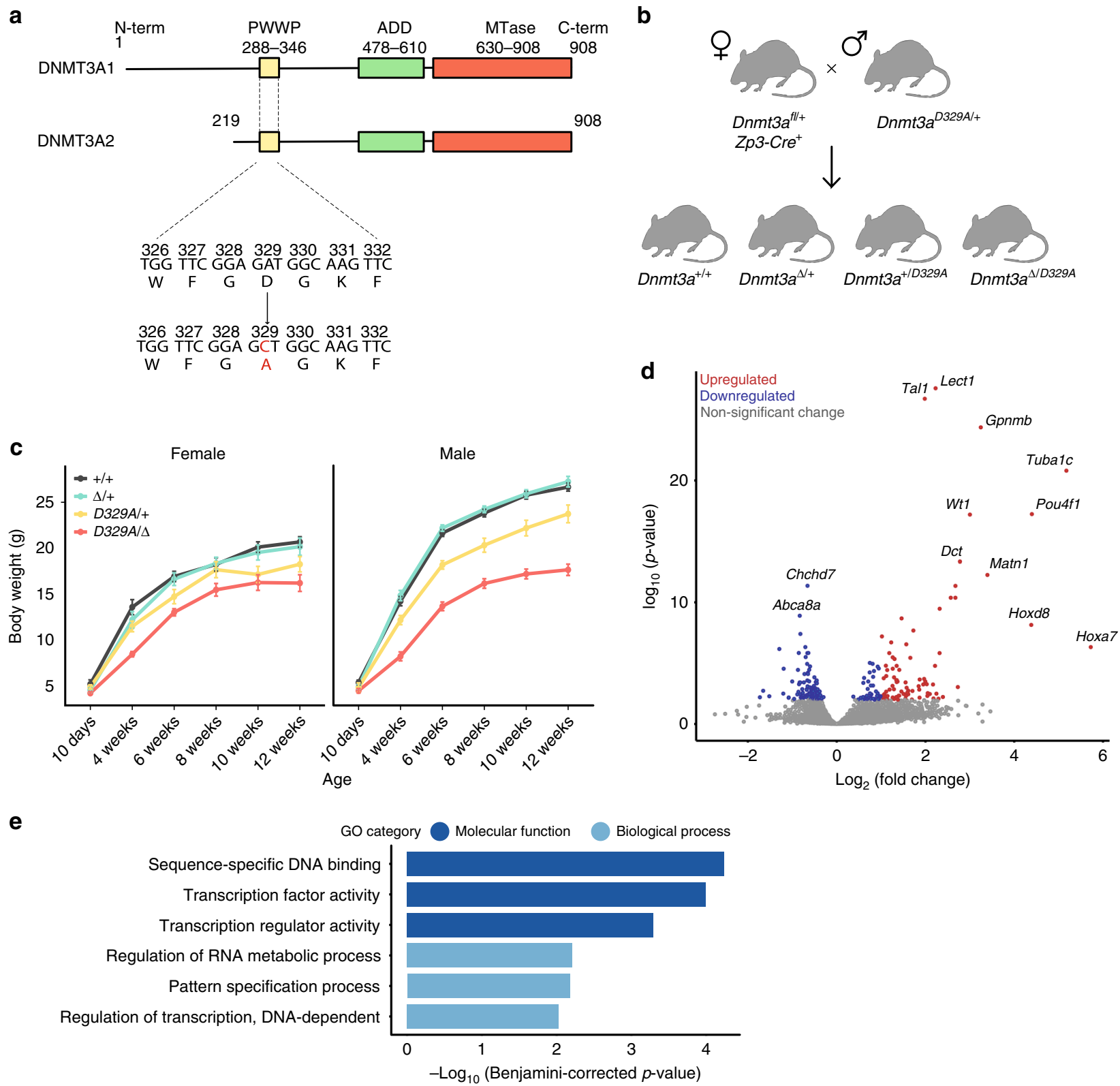

Fig. 1 Phenotypic description of mice carrying the Dnmt3aD329A allele. a Schematic representation of the DNMT3A protein isoforms and location of the base substitution, coloured red, introduced to generate the D329A mutation. NCBI IDs: CCDS36397.1, CCDS25784.1. Known domains are indicated by coloured boxes, based on the PROSITE database; numbers indicate amino acid residues. $\mathbf{b}$ Breeding scheme used to generate offspring of four different genotypes. +; wild-type allele; fl: Dnmt3a containing loxP sites surrounding exon 18; $\Delta$ : loxP sites after recombination leading to deletion of exon 18; Zp3-Cre: oocyte-specific Zp3 promoter-driven Cre recombinase ${ }^{21,22}$; D329A: a Dnmt3a allele point missense mutation in exon 8. c Growth curves indicating change in body weight of male and female mice of different genotypes over the indicated post-natal weeks. Mixed model ANOVA (KR-method) shows overall significant change dependent on genotype and time in both sexes $(p<0.001$, $\mathrm{df}$ (males) $=15,229.48$, $\mathrm{df}(\mathrm{females})=15,142.15), F=0)$. Subsequent pairwise Bonferroni-adjusted $t$-test comparisons show that Dnmt3a $a^{\Delta / D 329 A}$ males become significantly underweight at week $4(p<0.001)$, and Dnmt3a+/D329A at week $6(p<0.001)$. Dnmt3a $a^{4 / D 392 A}$ females become significantly underweight starting from week $4(p=0.002)$, with the exception of week $8(p=0.095)$. Dnmt3a+/D329A females do not show significant change. $n$ values are given in Supplementary Table 3. Error bars indicate standard error of the mean. Raw data are provided in Source Data. d Volcano plot showing gene expression fold change and its significance between Dnmt3a+/+ and Dnmt3a $/ / D 329 A$ adult (14-week) hypothalamus. Differentially expressed genes were determined using DEseq $(p<0.01$, Benjamini-Hochberg multiple comparisons correction). $n(+/+)=5, n(\Delta / D 392 A)=4$. Selected genes showing the most significant and highest fold change are named. e Gene ontology (GO) analysis of differentially expressed genes; only significant terms are shown. Benjamini-Hochberg corrected $p$-value $<0.1$ was used as a threshold

Transcriptional de-repression in $D n m t 3 a^{\Delta / D 329 A}$ hypothalamus. DNMT3A plays a predominant role in de novo methylation during postnatal neurogenesis ${ }^{23,51}$ and the hypothalamus is involved in regulation of body weight through endocrine function, feeding behaviour and energy homoeostasis ${ }^{52,53}$. We therefore evaluated potential gene dysregulation by performing RNA-seq on adult (14-week) hypothalamus. The D329A allele did not cause a statistically significant change in Dnmt3a transcript levels, or affect the abundance of other Dnmt or Tet transcripts (Supplementary Fig. 3). However, we identified 259 differentially expressed genes (DEGs) between Dnmt $3 a^{\Delta / D 329 A}$ and Dnmt $3 a^{+/+}$ (Fig. 1d and Supplementary Data 1). Gene ontology (GO) analysis 
indicated that upregulated genes were enriched in transcriptional regulators (Fig. 1e and Supplementary Data 2). Although downregulated genes did not show any significant GO term enrichment, amongst them were Ghsr, Bdnf, Sirt1 and Cartpt, key hypothalamic regulators of feeding and energy expenditure ${ }^{54-57}$. More striking, however, was the observation that a number of repressed transcription factor genes, such as Tal1, Pou4f1, Wt1, Hoxa7 and Hoxd8, showed dramatic upregulation in the $D n m t 3 a^{\Delta / D 329 A}$ hypothalamus (Fig. 1d). While growth retardation in the mutants could result from altered expression of the aforementioned metabolic regulators or transcription factors, it is important to highlight that, unusually, the most significant transcriptional changes were observed among genes that are normally deeply repressed in adult tissues.

The DNMT3A $\mathrm{A}^{\mathrm{D29A}}$ mutant causes aberrant gain of methylation. To determine whether changes observed in gene expression were due to aberrant DNA methylation, we performed genomewide DNA methylation profiling in adult (14-week) hypothalamus. Although the global DNA methylation level was similar across the four genotypes, with mean levels of $77.2 \%, 75.1 \%$, $77.2 \%$, and $75 \%$ in $D n m t 3 a^{+/+}, D n m t 3 a^{\Delta /+}, D n m t 3 a^{+/ D 329 A}$, and Dnmt $3 a^{\Delta / D 329 A}$, respectively, they could be separated by principal component analysis (Fig. 2a, Supplementary Fig. 4a, b). Individual samples clustered together into groups based on genotype, while genotype groups separated spatially, indicating high reproducibility between animals of each genotype, but distinct global methylation patterns between genotypes. The slight reduction in methylation in $D n m t 3 a^{\Delta /+}$ in comparison to Dnmt $3 a^{+/+}$suggested haploinsufficiency at a molecular level. A similar effect was observed between Dnmt3a+/D329A and $D n m t 3 a^{\Delta / D 329 A}$, suggesting that DNMT3A dosage is important for $\sim 2 \%$ of genome-wide DNA methylation levels in this tissue.

As gene bodies are normally marked by $\mathrm{H} 3 \mathrm{~K} 36 \mathrm{me} 3$, the putative target of the DNMT3A-PWWP domain, we first assessed methylation over gene bodies. All genes were highly methylated irrespective of expression level (Supplementary Fig. 4c), demonstrating that the Dnmt $3 a^{D 329 A}$ mutation does not profoundly affect gene body DNA methylation, although a similar haploinsufficiency effect to that seen in global methylation was observed in comparing the four genotypes. This effect extended to promoter regions, but for $\mathrm{CpG}$ island (CGI) containing promoters the trend was reversed, with higher levels of DNA methylation in the presence of the Dnmt $3 a^{D 329 A}$ allele (Supplementary Fig. 4d). Surprised by this finding, we used the EdgeR tool to compare Dnmt3a $a^{+/+}$and Dnmt3a $a^{\Delta / D 329 A}$, which identified 4184 statistically differentially methylated regions (DMRs) $(p$ (adj) $<0.01)$. Strikingly, the majority of DMRs $(3584$; $85.7 \%)$ showed gain of DNA methylation in Dnmt3a $a^{\Delta / D 329 A}$ compared to Dnmt3a $+1+$ (Fig. 2b-e Supplementary Figs. 5, 6 and Supplementary Data 3). To discriminate the effects of haploinsufficiency versus the D329A mutation, and whether hypomethylated domains were a result of the mutation, we used the same statistical tool to identify DMRs between $D n m t 3 a^{\Delta /+}$ and $D n m t 3 a^{\Delta / D 329 A}$, and between Dnmt $3 a^{+/+}$and Dnmt $3 a^{\Delta /+}$. In the Dnmt $3 a^{\Delta /+}$ and Dnmt $3 a^{\Delta / D 329 A}$ comparison, which corrects for the number of alleles, only 75 out of 4603 DMRs were hypomethylated, whereas in the Dnmt3a $a^{+/+}$and $D n m t 3 a^{\Delta /+}$ comparison, 328 out of 351 DMRs were hypomethylated (Fig. 2f, $\mathrm{g}$ and Supplementary Data 4 and 5). This demonstrates that hypomethylation was primarily a result of haploinsufficiency, whilst hypermethylation was caused by the DNMT3A ${ }^{\mathrm{D} 329 \mathrm{~A}}$ mutation.

Analysis of DNA methylation of DEGs showed that only about one in five misregulated genes were associated with DNA methylation changes (Supplementary Figs. 7-9), suggesting a substantial proportion of DEGs may be attributable to indirect, downstream effects of direct targets. A cluster of genes that showed a gain of DNA methylation over both promoter and gene body stood out in the analysis (Supplementary Fig. 7). Many of these were the same developmental transcription factor genes that are normally strongly repressed in adult hypothalamus, but which are paradoxically derepressed in Dnmt $3 a^{\Delta / D 329 A}$ (e.g., Hoxa7, Hoxd8, Pou4f1, Tal1, and Wt1). It is important to note that despite the increase in DNA methylation over these genes, their transcription start sites remained relatively less methylated than the surrounding regions (Supplementary Fig. 10).

Taken together, these findings suggest that the Dnmt3a $a^{D 29 A}$ allele is a driver of aberrant gain of DNA methylation and derepression of a subset of associated genes; consequently, all subsequent analyses are focussed on hypermethylated DMRs.

DNA methylation alterations occur in multiple tissues. To assess whether the observed DNA methylation abnormalities were tissue-specific, we also analysed DNA methylation in adult (14-week) pituitary and liver. We chose pituitary because of its functional link with hypothalamus in growth regulation ${ }^{58}$ and liver as an unrelated and relatively homogeneous tissue. As with hypothalamus, the four Dnmt3a genotypes had similar global DNA methylation levels in these tissues, but could be separated by principal component analysis (Fig. 3a, b). Using the EdgeR tool to identify DMRs, both liver and pituitary showed an excess of hypermethylated sites similar to, although to a lesser extent than, the hypothalamus (Supplementary Data 6-9). Moreover, hypermethylation in both tissues was specifically related to the presence of the D329A allele (Fig. 3c, d). Strikingly, there was substantial overlap of DMRs between the three tissues, particularly amongst hypermethylated probes (Fig. 3e). The occurrence of shared hypermethylated DMRs, albeit with tissue-specific differences in magnitude, raised the question of whether aberrant DNA methylation arose early in embryogenesis prior to cell lineage specification or independently in each tissue.

DNA methylation gain is progressive in postnatal development. The major phase of DNA methylation establishment in mice occurs during early post-implantation development $(\mathrm{E} 4.5-\mathrm{E} 6.5)^{5,59}$. To address the possibility that aberrant methylation was established during this phase of embryonic remethylation, we collected E7.5 mouse epiblasts (Epi) from embryos of all four genotypes. Global methylation was more variable in Dnmt $3 a^{+/ D 329 A}$ and Dnmt $3 a^{\Delta / D 329 A}$ embryos than Dnmt3a $a^{+/+}$ and $D n m t 3 a^{\Delta /+}$ (Supplementary Fig. 11). Overall, methylation in $D n m t 3 a^{+/ D 329 A}$ and Dnmt $3 a^{\Delta / D 329 A}$ embryos was lower than wild-type at all genomic features; however, at CGI promoters this may be attributable to a haploinsufficiency effect as reduced DNA methylation was also observed in Dnmt $3 a^{\Delta /+}$ embryos. We used EdgeR to assess Dnmt $3 a^{+/+}$and $D n m t 3 a^{\Delta /+}$ embryos and identified 136 DMRs out of a total of 108,212 tiles (0.13\%; Supplementary Data 10). All but two DMRs were overlapping a CGI, again pointing to the potential role of DNMT3A in CGI methylation during early development. Comparison between Dnmt $3 a^{+/+}$and Dnmt3a $a^{\Delta / D 329 A}$ embryos identified only five significantly hypomethylated tiles out of a total of 108,212 (0.005\%; Supplementary Data 11). This suggests that the presence of mutant DNMT3A results in stochastic delay in de novo methylation across genomic regions rather than any locus specificity. In agreement with this, embryos did not cluster based on genotype (Supplementary Fig. 11b-d). Crucially, there was no evidence of hypermethylation in Dnmt $3 a^{\Delta / D 329 A}$ mutants at E7.5 (Supplementary Fig. 11e-j), suggesting that it was not set during 
a

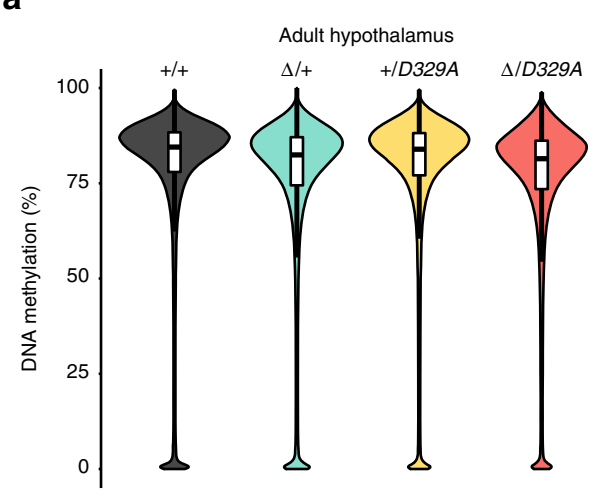

C

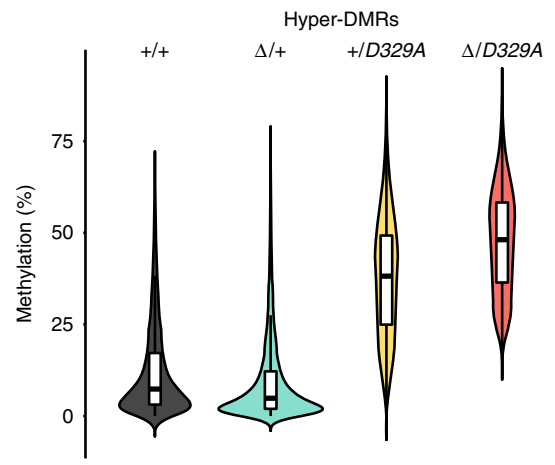

b

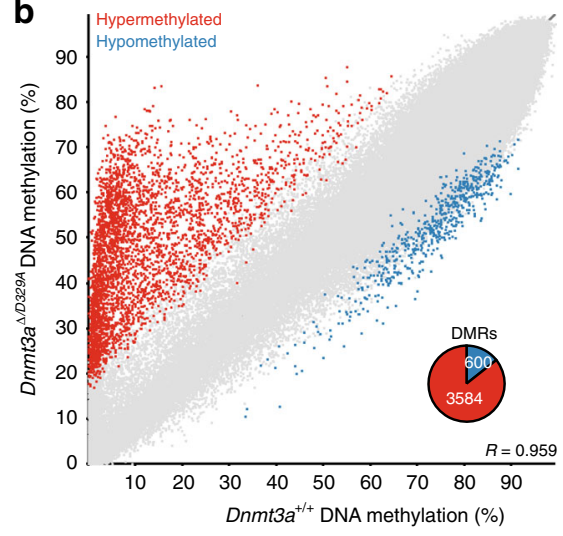

d

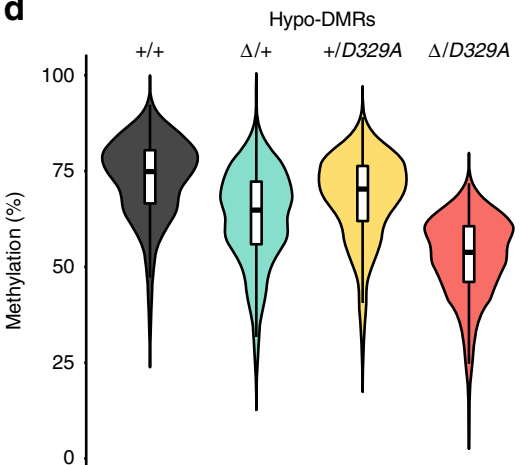

e

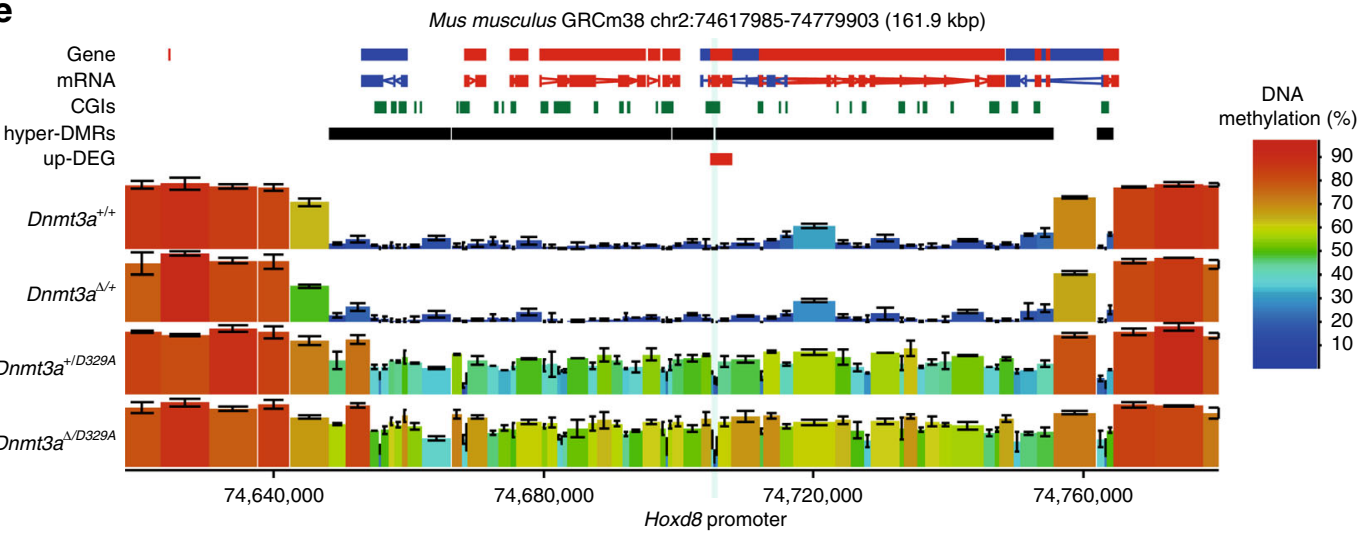

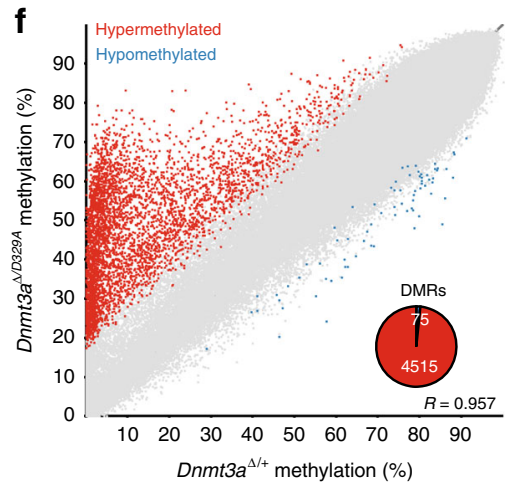

the major wave of post-implantation de novo methylation. In view of this result, and because hypothalamic neurons undergo further epigenetic modelling during postnatal maturation ${ }^{51}$, we next assessed methylation in hypothalamus at postnatal days 1

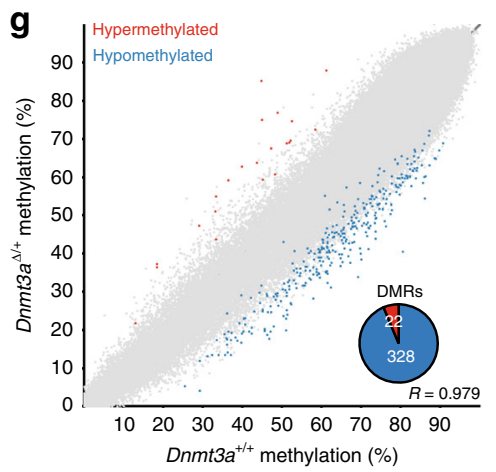

and 25 (P1 and P25). This showed a progressive gain of DNA methylation across the domains destined to be hypermethylated in Dnmt $3 a^{\Delta / D 329 A}$ adults (Fig. 4a-d). Together, these findings suggest that the aberrant gain of DNA methylation caused by the 
Fig. 2 DNA methylation changes in mice carrying Dnmt3a $329 \mathrm{~A}$ allele. a Beanplots indicating whole genome methylation levels in hypothalamus of adult (14 week, male) mice carrying the alleles shown. Boxplot shows median value and 25-75th percentiles, whiskers show lowest and highest observation, excluding outliers. Raw data are provided in Source Data. b Scatterplot showing correlation of methylation levels of individual tiles between Dnmt3a $+/+$ and Dnmt3a $a^{\Delta / D 329 A}$ mice. Differentially methylated probes were determined using the EdgeR proportion statistic in SeqMonk ( $p<0.01$ corrected for multiple comparisons using Benjamini-Hochberg, methylation difference $\geq 20 \%$ ). Pie-chart indicates how many of DMRs are hyper-methylated or hypo-methylated in Dnmt3as/D329A. $n=3$ for each genotype. c, d Beanplots indicating DNA methylation levels over hypermethylated (c) and hypomethylated (d) DMRs. Boxplot shows median value and 25-75th percentiles, whiskers show lowest and highest observation, excluding outliers. Source data are listed in

Supplementary Data 3. e Representative genome browser region showing the Hoxd hypermethylated domain. De-repressed gene Hoxd8 promoter region is shaded. For gene and mRNA tracks, the colour indicates direction, where red is a forward strand and blue is a reverse strand. Colour-coded blocks are tiles of 100-CpG positions. Error bars indicate standard deviation. $\mathbf{f}, \mathbf{g}$ Scatterplots showing correlation between methylation levels of 100-CpG tiles in 14-week adult male hypothalamus of $\mathbf{f} D n m t 3 a^{\Delta /+}$ and Dnmt $3 a^{\Delta / D 329 A}$, and $\mathbf{g} D n m t 3 a^{+/+}$and Dnmt $3 a^{\Delta /+}$. Differentially methylated tiles were determined using the EdgeR proportion statistic in SeqMonk ( $p<0.01$ corrected for multiple comparisons using Benjamini-Hochberg, methylation difference $\geq 20 \%$ ). The piechart indicates the number of DMRs identified. In a-g, Hypo: hypomethylated, hyper: hypermethylated. Tiles of 100-CpG positions. $n(+/+, \Delta /+, \Delta /$ $D 329 A)=3, n(+/ D 329 A)=2$. CGI: CpG island, DMR: differentially methylated region

D329A allele occurs in tissues independently during postnatal development rather than early development.

Hypermethylated loci are enriched in transcription factors. To identify what was shared amongst loci anomalously methylated by DNMT3 $\mathrm{A}^{\mathrm{D} 329 \mathrm{~A}}$, we evaluated their genomic properties. As previously observed, hypermethylated DMRs were strongly enriched in CGIs (Fig. 5a). We also noticed that many hypermethylated DMRs fell within large normally unmethylated genomic regions (Fig. 2e), features previously described as methylation 'valleys' or 'canyons' that are conserved amongst vertebrates ${ }^{60,61}$. Of the 1104 canyons originally defined in hematopoietic stem cells ${ }^{60}, 779$ were also hypomethylated in wild-type hypothalamus (Supplementary Data 12) and displayed a substantial increase in DNA methylation in Dnmt3a $a^{\Delta / D 329 A}$ (Fig. 5b). GO analysis indicated that genes within hypermethylated DMRs in Dnmt3a $a^{\Delta / D 329 A}$ hypothalamus were strongly enriched in transcription factors and regulators of morphogenesis and differentiation (Fig. 5c and Supplementary Data 13).

Gain of DNA methylation is linked to H3K27me3. Developmental transcription factor genes that showed dysregulated expression and DNA methylation are embedded in methylation canyons typically marked by bivalent chromatin and silenced by Polycomb repressor complexes ${ }^{60,62,63}$. Thus, we hypothesised that DNMT3 $\mathrm{A}^{\mathrm{D} 329 \mathrm{~A}}$ hypermethylated DMRs would be enriched in $\mathrm{H} 3 \mathrm{~K} 4 \mathrm{me} 3$ and $\mathrm{H} 3 \mathrm{~K} 27 \mathrm{me} 3$. To assess this, we generated $\mathrm{H} 3 \mathrm{~K} 4 \mathrm{me} 3, \mathrm{H} 3 \mathrm{~K} 27 \mathrm{me} 3$, and $\mathrm{H} 3 \mathrm{~K} 36 \mathrm{me} 3$ chromatin immunoprecipitation-sequencing (ChIP-seq) datasets from adult (14-week) hypothalamus. Overall, these histone marks exhibited strong correlation between Dnmt3a $a^{+/+}$and Dnmt $3 a^{\Delta / D 329 A}$ samples (Supplementary Fig. 12), indicating no marked shift in the genomic distribution of these marks in the presence of the Dnmt $3 a^{D 329 A}$ allele. Strikingly, however, whereas hypermethylated and hypomethylated DMRs were not obviously separated in terms of enrichment levels for $\mathrm{H} 3 \mathrm{~K} 4 \mathrm{me} 3$ or $\mathrm{H} 3 \mathrm{~K} 36 \mathrm{me} 3$, there was a clear separation in their enrichment for H3K27me3 (Fig. 6a-c).

To explore this effect more rigorously, we called H3K36me3, $\mathrm{H} 3 \mathrm{~K} 27 \mathrm{me} 3$, and $\mathrm{H} 3 \mathrm{~K} 4 \mathrm{me} 3$ peaks and observed that $70.4 \%$ of hypermethylated DMRs were bivalent, i.e., contained both H3K27me3 and H3K4me3 peaks, with an additional $26.3 \%$ containing H3K27me3 only peaks (Fig. 6d), suggesting that DNMT3A ${ }^{\mathrm{D} 329 \mathrm{~A}}$ is preferentially recruited to H3K27me3-marked chromatin and is able to methylate bivalent DNA marked by $\mathrm{H} 3 \mathrm{~K} 4 \mathrm{me} 3$ in the presence of $\mathrm{H} 3 \mathrm{~K} 27 \mathrm{me} 3$. In contrast, only 11 (1.8\%) hypomethylated and 74 (2.1\%) hypermethylated domains overlapped $\mathrm{H} 3 \mathrm{~K} 36 \mathrm{me} 3$ peaks, again indicating that DMRs were not associated with this histone mark. We then sought to discriminate between two possible mechanisms to explain the aberrant targeting of DNMT3A $\mathrm{A}^{\mathrm{D} 29 \mathrm{~A}}$ to Polycomb-associated methylation canyons: (1) the D329A mutation leads to promiscuous localisation and activity of DNMT3A due to a loss of association with H3K36me3-marked chromatin; or (2) DNMT3A $^{\text {D29A }}$ is selectively recruited to $\mathrm{H} 3 \mathrm{~K} 27 \mathrm{me} 3$-marked chromatin. To do this, we looked first at DNA methylation at gene bodies with low (bottom quintile) or high (top quintile) levels of H3K36me3. In a comparison of the four genotypes, we observed no significant differences in gene body DNA methylation associated with the Dnmt $3 a^{D 329 A}$ allele (Fig. 6e). We then selected regions that are hypomethylated in Dnmt $3 a^{+/+}$ hypothalamus (methylation level below $10 \%$; $4.6 \%$ of the genome; Fig. $6 \mathrm{f}$ ) and partitioned hypomethylated probes based on low or high levels of enrichment of $\mathrm{H} 3 \mathrm{~K} 4 \mathrm{me} 3$, H3K27me3, or H3K36me3. By this analysis, neither the presence nor absence of either $\mathrm{H} 3 \mathrm{~K} 4 \mathrm{me} 3$ or $\mathrm{H} 3 \mathrm{~K} 36 \mathrm{me} 3$ were associated with DNA hypermethylation by DNMT3A $329 \mathrm{~A}$ (Fig. 6g, h). In contrast, hypomethylated regions highly enriched in $\mathrm{H} 3 \mathrm{~K} 27 \mathrm{me} 3$ were preferentially hypermethylated (Fig. 6i). This suggests that hypomethylation of genomic domains per se is not sufficient to recruit and activate $\mathrm{DNMT} 3 \mathrm{~A}^{\mathrm{D} 329 \mathrm{~A}}$. Furthermore, bivalent regions marked by both $\mathrm{H} 3 \mathrm{~K} 27 \mathrm{me} 3$ and $\mathrm{H} 3 \mathrm{~K} 4 \mathrm{me} 3$ also showed a substantial gain in DNA methylation (Fig. 6i), although to a lesser degree than $\mathrm{H} 3 \mathrm{~K} 27 \mathrm{me} 3$ alone, suggesting the presence of $\mathrm{H} 3 \mathrm{~K} 4 \mathrm{me} 3$ remains antagonistic to DNMT3A $\mathrm{D}^{\mathrm{D} 29 \mathrm{~A}}$ activity. Together these results support the conclusion that DNMT3A $\mathrm{A}^{\mathrm{D} 329 \mathrm{~A}}$ is selectively recruited to $\mathrm{H} 3 \mathrm{~K} 27 \mathrm{me} 3$-marked chromatin.

Because H3K27me3 and DNA methylation show mutual exclusivity in some genomic contexts ${ }^{64-66}$, we were interested whether the histone landscape would be altered, with DNA methylation replacing $\mathrm{H} 3 \mathrm{~K} 27 \mathrm{me} 3$ at hypermethylated DMRs. Although the levels of DNA methylation gain were marginally associated with loss of $\mathrm{H} 3 \mathrm{~K} 27 \mathrm{me} 3$ (Fig. 6j), these marks appeared to co-exist with hypermethylated domains retaining a substantial amount of $\mathrm{H} 3 \mathrm{~K} 27 \mathrm{me} 3$, even at CGIs, and we observed that the extent of DNA methylation gain correlated better with initial enrichment of $\mathrm{H} 3 \mathrm{~K} 27 \mathrm{me} 3$ over the locus than its loss (Fig. 6k). Finally, we were able to use the ChIP-seq data to evaluate that for a subset of DEGs there were associated changes in promoter $\mathrm{H} 3 \mathrm{~K} 4 \mathrm{me} 3$ (Fig. 61). These findings support the link between $\mathrm{H} 3 \mathrm{~K} 27 \mathrm{me} 3$ and abnormal localisation of DNMT3A $\mathrm{A}^{\mathrm{D} 329 \mathrm{~A}}$, and suggest that the normal histone landscape is affected by the aberrant gain in DNA methylation (Fig. 7).

Non-CpG methylation shows altered DNMT3A $\mathrm{A}^{\mathrm{D} 329 \mathrm{~A}}$ recruitment. Non-CpG $(\mathrm{CpH})$ methylation becomes particularly abundant in neural tissues, where it is established postnatally by 

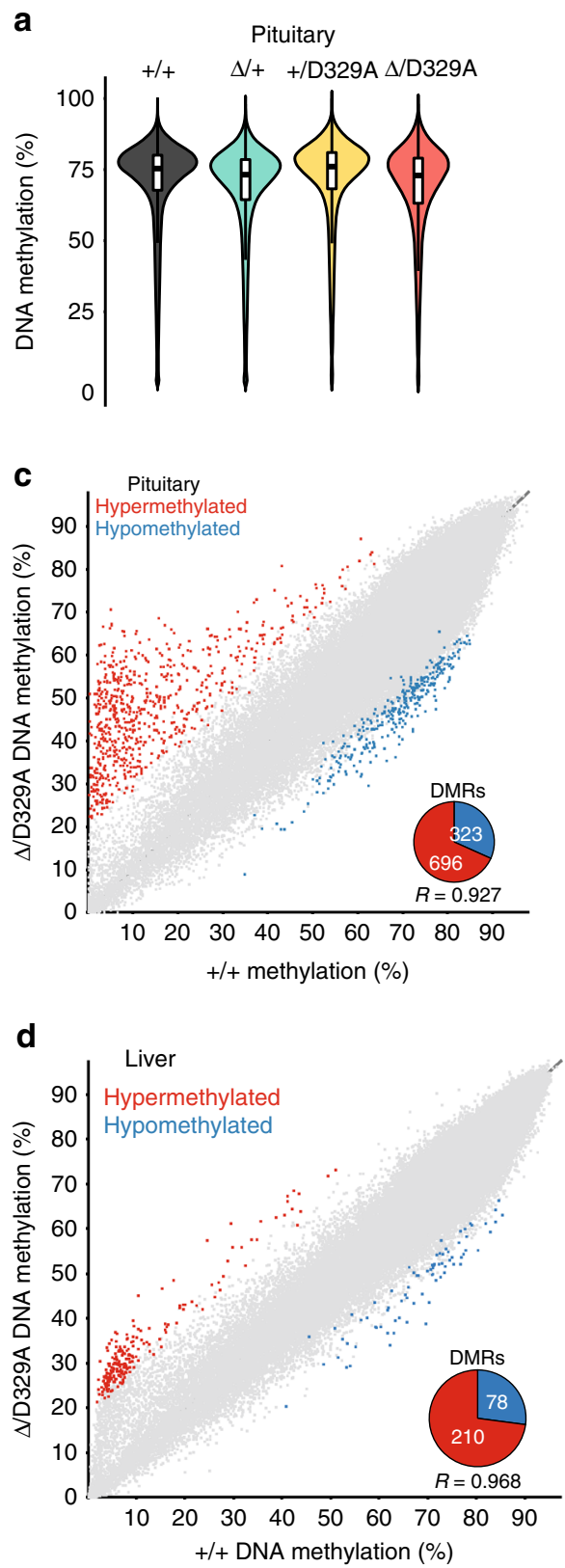
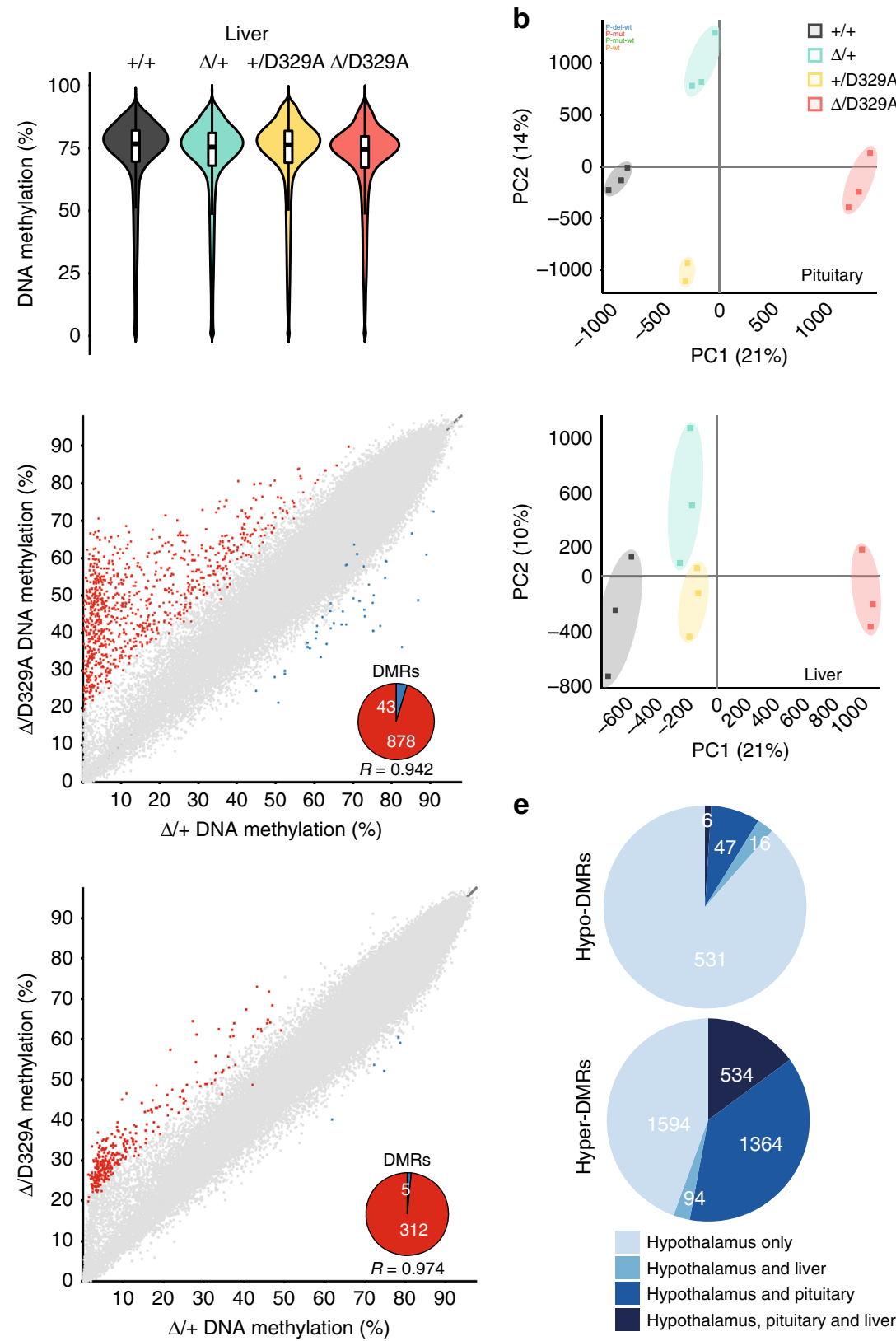

Fig. 3 DNA methylation in adult pituitary and liver. a Beanplots indicating whole genome methylation levels in pituitary and liver of 14-week adult male mice carrying the alleles shown. Tiles of $300 \mathrm{CpG}$ positions. Boxplot shows median value and 25-75th percentiles, whiskers show lowest and highest observation, excluding outliers. Raw data are provided in Source Data. b PCA plots showing clustering of individual pituitary and liver samples into separate distant groups based on the genotype. c, d Scatterplots showing correlation between methylation levels of individual 300-CpG tiles in 14-week adult male (c) pituitary and (d) liver between Dnmt3a+/+ and Dnmt3a $a^{\Delta / D 329 A}$, and between Dnmt3a $a^{\Delta /+}$ and Dnmt3a $a^{\Delta / D 329 A}$. Differentially methylated tiles were determined using the EdgeR proportion statistic in SeqMonk $(p<0.01$ corrected for multiple comparisons using Benjamini-Hochberg, methylation difference $\geq 20 \%$ ). Pie-chart insets indicate how many of DMRs are hyper-methylated or hypo-methylated. e Pie charts showing how many hypomethylated and hyper-methylated tiles in hypothalamus overlap the corresponding differentially methylated tiles in pituitary, liver or both. Pituitary and liver: tiles of 300-CpG position; hypothalamus: tiles of 100-CpG positions. In a-d, $n$ (pituitary, $+/+, \Delta /+, \Delta / D 329 A)=3, n($ pituitary, $+/ D 329 A)=2$, $n($ liver $)=3$

DNMT3A $^{67-69}$. Importantly, $\mathrm{CpH}$ methylation is not maintained at DNA replication, so its presence is a record of de novo methylation since the last replication event. Thus, we investigated $\mathrm{CpH}$ methylation in adult hypothalamus as a read-out of DNMT3A localisation and activity (Fig. 8a). CpH methylation levels showed strong evidence for haploinsufficiency at the molecular level, as we observed very similar decreases in global levels in Dnmt $3 a^{\Delta /+}$ compared to Dnmt3a $a^{+/+}$, and Dnmt3a $a^{\Delta / D 329 A}$ compared to Dnmt3a+/D329A (Fig. 8a, b).
The smaller global reduction between $D n m t 3 a^{+/ D 329 A}$ and Dnmt $3 a^{+/+}$and between Dnmt3a $a^{\Delta / D 329 A}$ and Dnmt3a $a^{\Delta /+}$ suggests some decrease in methyltransferase activity by the mutant protein. Strikingly, however, the pattern over hypermethylated DMRs was reversed, with Dnmt3a $a^{+/ D 329 A}$ and Dnmt3a $a^{\Delta / D 329 A}$ showing increased $\mathrm{CpH}$ methylation compared to Dnmt3a+/+ and Dnmt $3 a^{\Delta /+}$, respectively (Fig. 8a, c). Comparing relative $\mathrm{CpH}$ methylation across prenatal and postnatal development showed that this effect first becomes evident in the P1 hypothalamus 
a

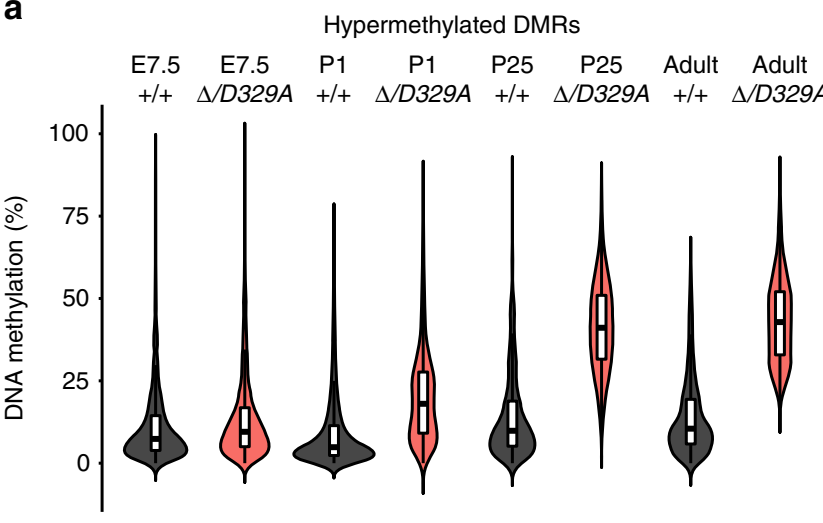

b

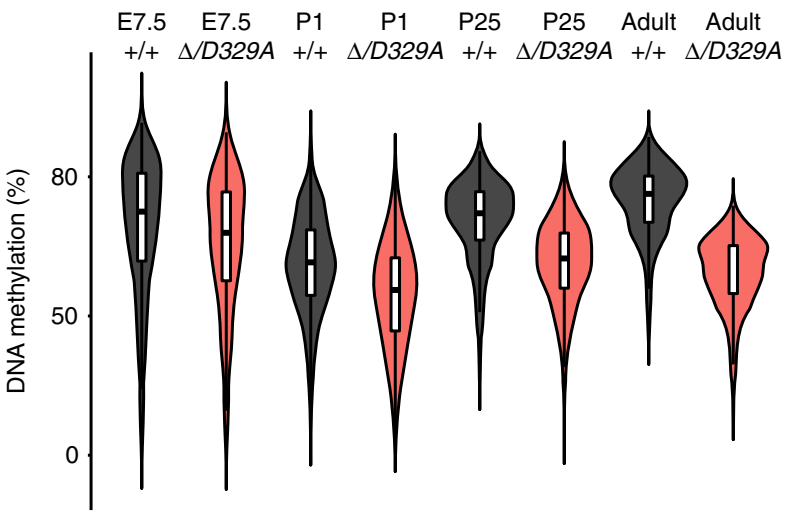
Hypomethylated DMRs
C

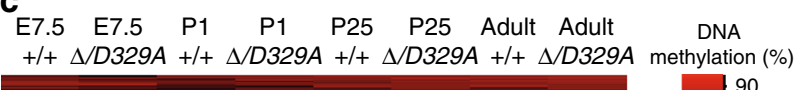

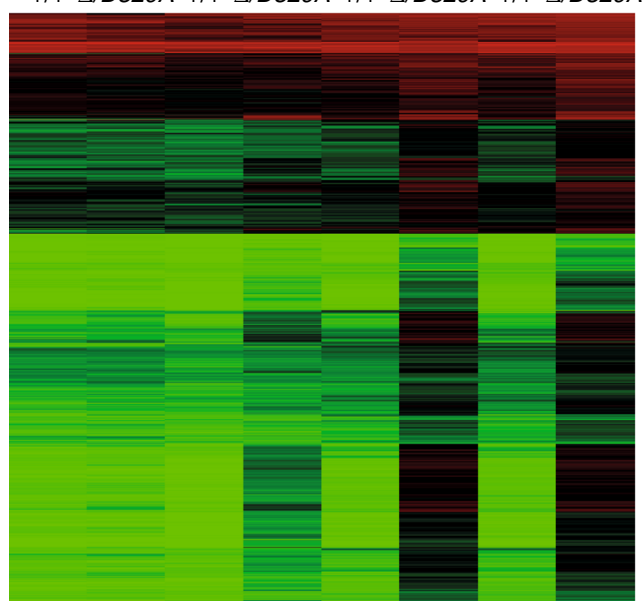

d 
a

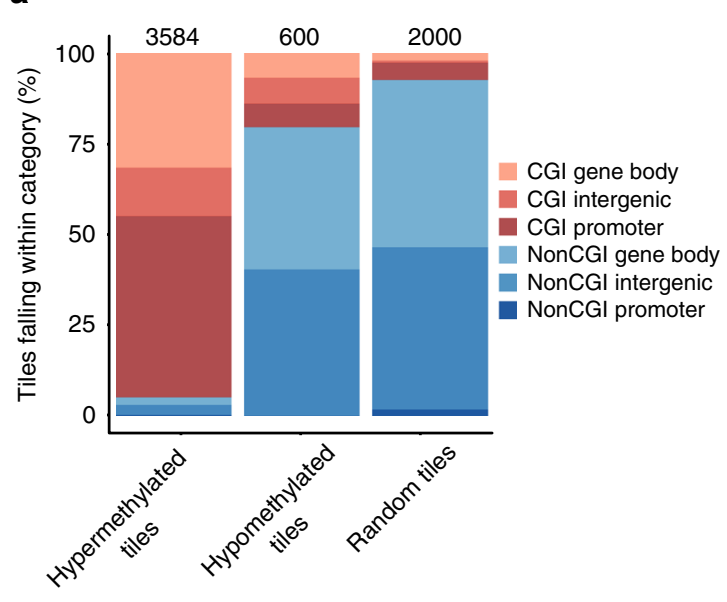

b

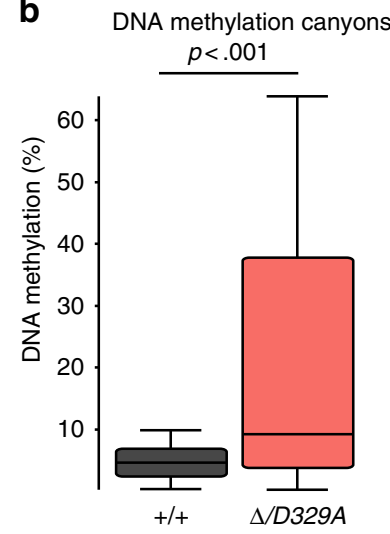

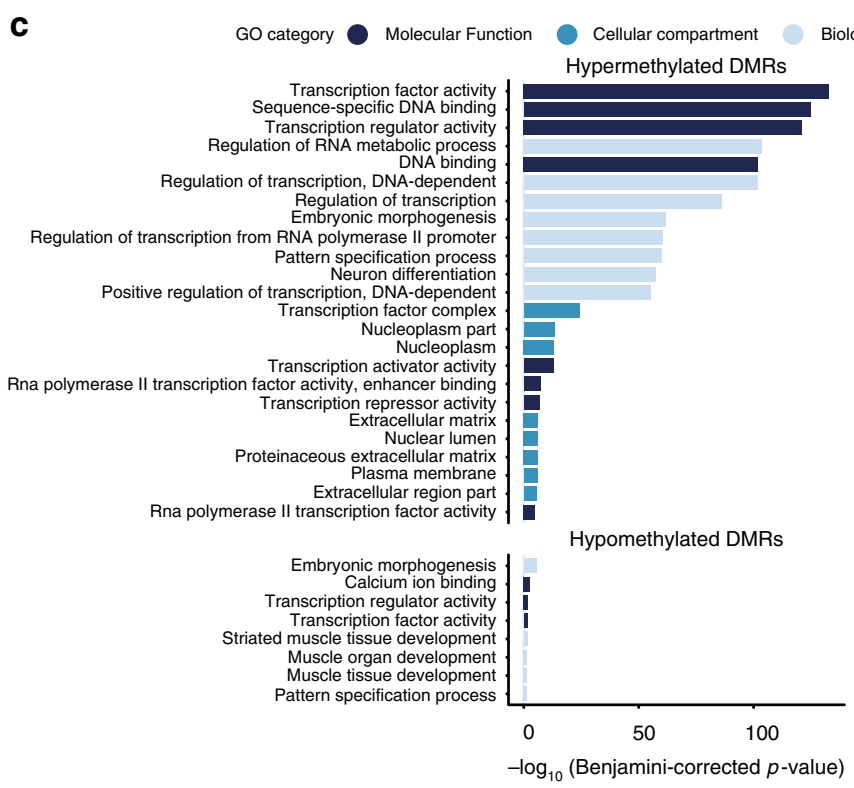

Fig. 5 Features of regions aberrantly methylated by DNMT3AD329A a Percentages of hypermethylated and hypomethylated tiles (100-CpG) falling within the genomic features indicated compared with a random set of 100-CpG tiles (Supplementary Data 14). $n$ (tiles) indicated on top of the bars. CGI: CpG island. b Distribution of methylation values over DNA methylation canyon probes 60 in Dnmt3a $/++$ and Dnmt3a $/ / D 329 A$ mice. $n=779$, Mann-Whitney test $\left(p<0.01, U_{A}=432,773, z=-14.57\right)$. Boxplots show median value and 25-75th percentiles, whiskers show lowest and highest observation, excluding outliers. Source data are provided in Supplementary Data 12. c GO analysis of genes overlapping differentially methylated tiles. Unless there were fewer significant terms, the top 8 most significant terms per category are shown. Benjamini-Hochberg corrected $p$-value $<0.01$ was used as a threshold. DMR differentially methylated region

methylation of genomic domains harbouring H3K27me3silenced developmental regulatory genes. We demonstrate that the gain of DNA methylation occurs specifically during postnatal development and is likely to be a consequence of an altered association of the mutant DNMT3A with chromatin. Furthermore, we observed transcriptional de-repression of a subset of the associated genes, suggesting the aberrant localisation of DNA methylation to these genomic regions paradoxically results in their activation.

The DNMT3A-PWWP domain has been described to bind to H3K36me2 and H3K36me3 in vitro, an interaction that was blocked by targeted mutations, including D $329 \mathrm{~A}^{27,44,47}$. In Dnmt3a $a^{D 29 A}$ E7.5 embryos, we observed a stochastic delay in de novo methylation that was not localised to particular genomic areas. In adult tissues, however, we did not observe a loss of DNA methylation attributable to the D329A mutation and find no evidence that this mutation alters DNA methylation levels at regions enriched for H3K36me3, suggesting the delays observed in embryonic development do not persist. Together, these findings suggest that the function of DNMT3A in vivo is not necessarily reliant on its association with H3K36me2/3, but that the D329A mutant protein may have reduced methylase activity due to an altered association with chromatin or catalytic activity. Subsequently, the delay in de novo methylation may be recovered with time or DNMT3B may be able to compensate during embryogenesis, supported by previous observations that DNMT3A and DNMT3B have partial redundancy in the postimplantation embryo ${ }^{18,50}$.

In addition to an interaction with $\mathrm{H} 3 \mathrm{~K} 36 \mathrm{me} / 3$, wild-type DNMT3A has been found to associate specifically with H3K27me3-marked shores of bivalent CGIs ${ }^{70,71}$. In our study, the DNMT3A $\mathrm{A}^{\mathrm{D} 329 \mathrm{~A}}$ mutant aberrantly methylates bivalent and H3K27me3-marked genomic domains in postnatal development. This hypermethylation is selective towards polycomb repressive complex (PRC2)-mediated H3K27me3 domains rather than 
a

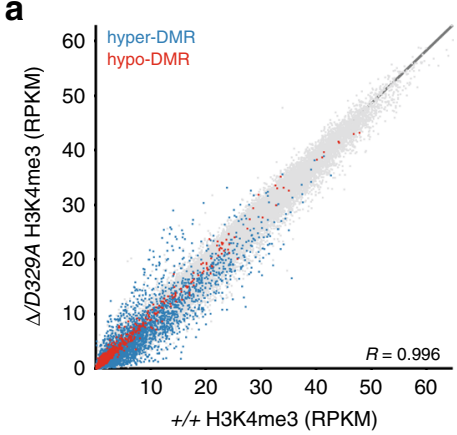

d

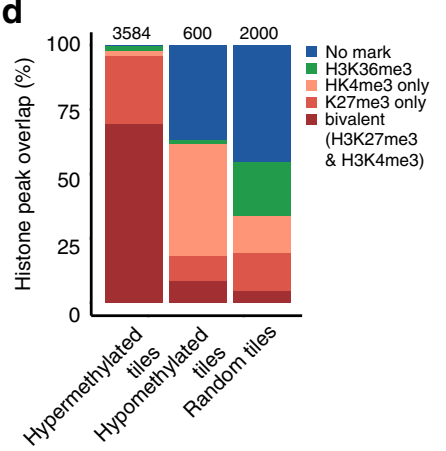

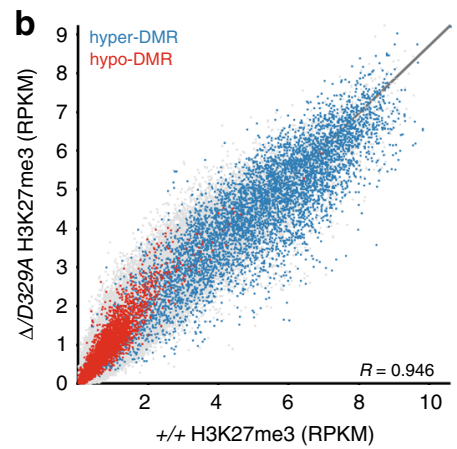

e

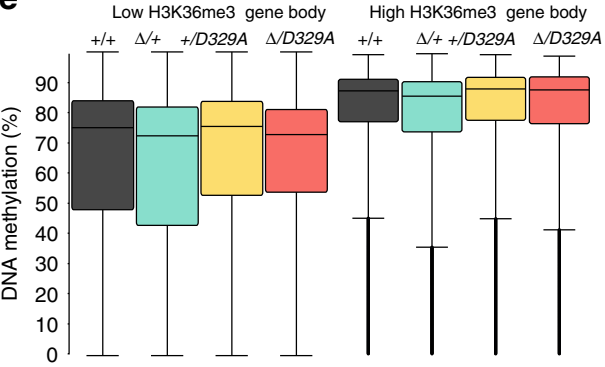

C

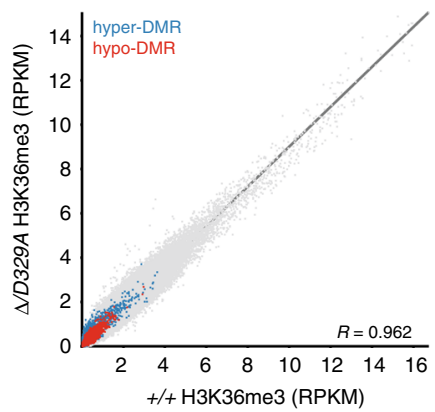

f

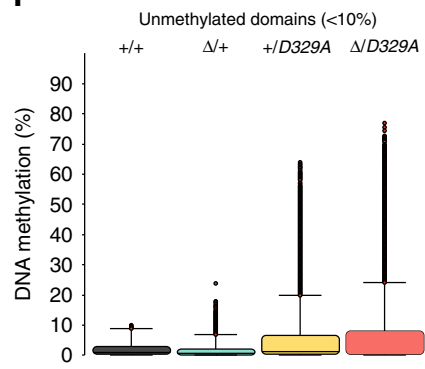

g

High H3K4me3 (excl. bivalent)

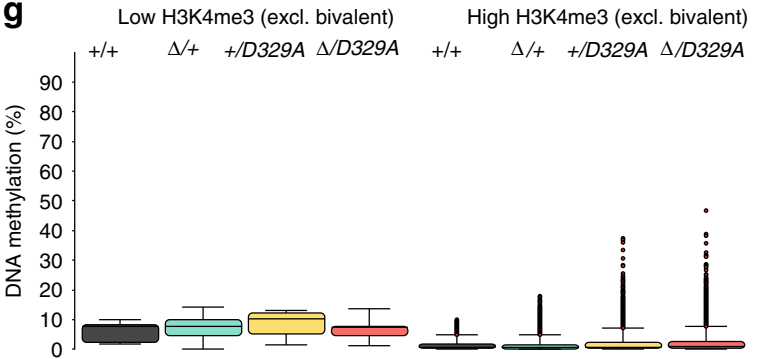

h

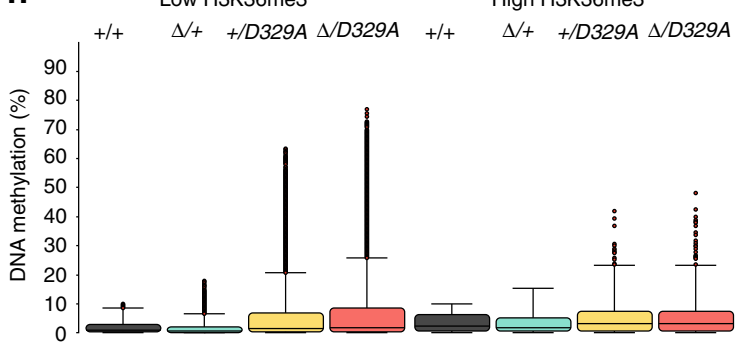

i

High H3K27me3 (excl. bivalent) bivalent (high H3K27me3 \& H3K4me3)

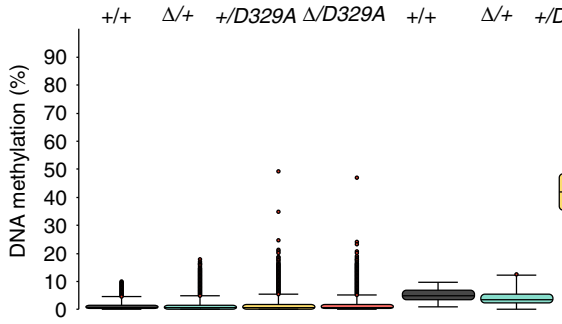

$27 \mathrm{me} 3$ (excl. bivalent)
$+\quad+/ D 329 A \Delta / D 329 A$

$+/+\quad \Delta /+\quad+/ D 329 A \Delta / D 329 A$
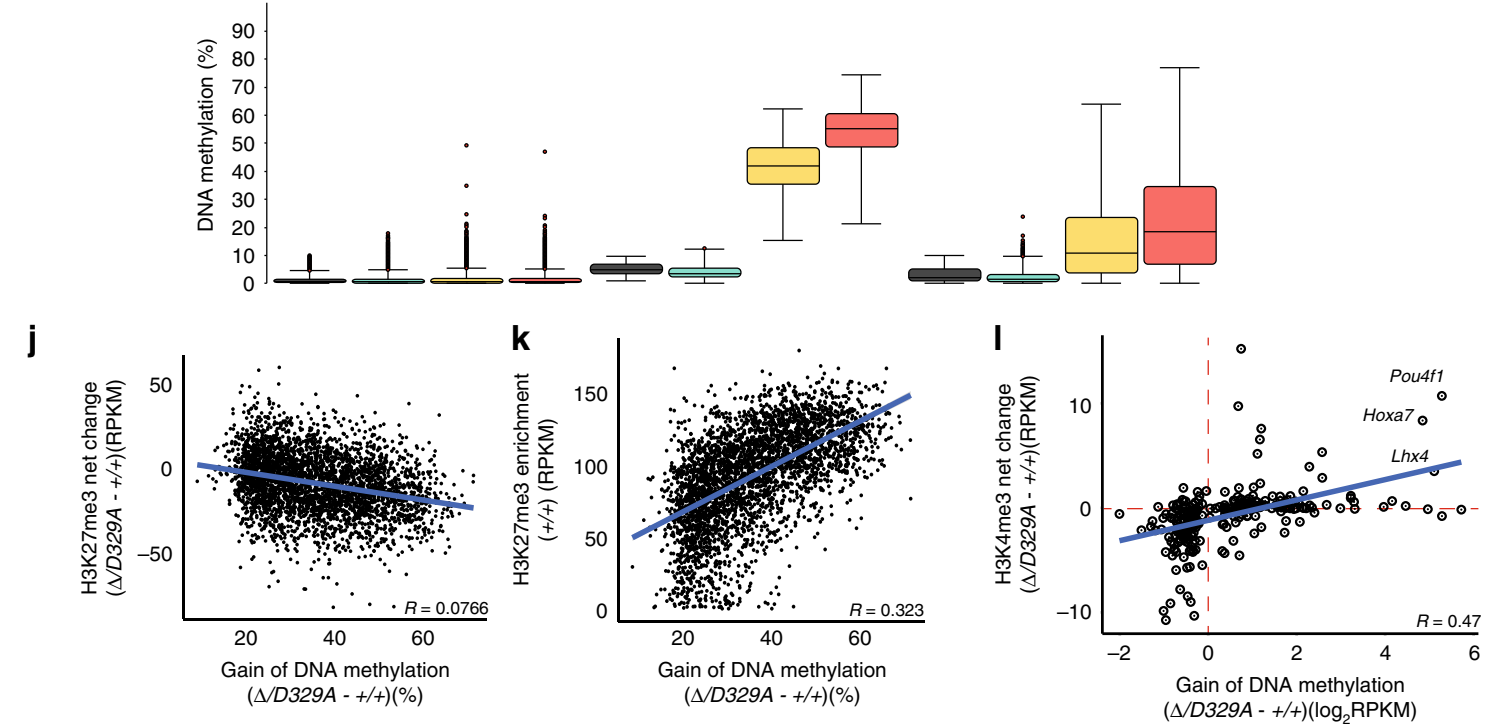

unmethylated regions of the genome in general, supporting an association between the PWWP domain and H3K27me3 in vivo ${ }^{27}$. Nonetheless, direct interaction evidence between DNMTs and H3K27me3 from biochemical experiments is limited $27,44,47,70$, and thus the nature of this association will need to be explored further.
DNA methylation and $\mathrm{H} 3 \mathrm{~K} 27 \mathrm{me} 3$ are repressive marks that have been widely reported to be mutually exclusive $67,72,73$. However, this effect is not observed genome-wide; it is largely restricted to regions containing CGIs ${ }^{66,74}$ and mediates the generation of large hypomethylated canyons or valleys across these CGI-rich domains, such as the Hox clusters ${ }^{60,61}$. The regulation 
Fig. 6 Link between $\mathrm{H} 3$ post-translational modifications and aberrant DNA methylation. a-c Scatterplots showing correlation between enrichments for a H3K4me3; b H3K27me3; c H3K36me3 between Dnmt3at/+ and Dnmt3a $a^{4 / D 329 A}$ hypothalamus, with the tiles overlapping hypermethylated (blue) and hypomethylated (red) DMRs indicated. $n=3$ each genotype. Tiles of $2 \mathrm{~kb}$ with a step of $1 \mathrm{~kb}$ were used for analyses. $\mathbf{d}$ Percentages of hypermethylated and hypomethylated DMRs (100-CpG) that overlap bivalent (H3K4me3 and H3K27me3), H3K27me3 only, H3K4me3 only and H3K36me3 peaks, or regions with no mark. A random set of 100-CpG tiles (excluding DMRs) was used as a whole genome representative (Supplementary Data 14). $n$ (tiles) indicated above each bar. H3K36me3 tiles that show overlap with H3K4me3 or H3K27me3 were included in the H3K36me3 group. Peaks were called using MACS peak caller in adult hypothalamus ChIP-seq. e DNA methylation levels over gene bodies marked by low or high levels of H3K36me3. DNA methylation quantified over individual gene bodies, excluding promoters. f DNA methylation levels across unmethylated genomic regions (<10\% DNA methylation in wild-type hypothalamus) amongst different genotypes. $\mathbf{g}$, h DNA methylation levels between genotypes over unmethylated regions overlapping low and high $\mathbf{g} \mathrm{H} 3 \mathrm{~K} 36 \mathrm{me} 3$ or $\mathbf{h} \mathrm{H} 3 \mathrm{~K} 4 \mathrm{me} 3$ marked chromatin. i DNA methylation levels between genotypes over unmethylated regions overlapping low, high H3K27me3, or both high H3K4me3 and high H3K27me3 (bivalent), marked chromatin. j Scatterplot showing the relationship between gain of DNA methylation in Dnmt3a $a^{4 / D 329 A}$ hypothalamus and change in enrichment of H3K27me3. Based on H3K27me3 enrichment difference over DMR tiles between $D n m t 3 a^{+/+}$and Dnmt3a $\Delta / D 329 A$ adult hypothalamus. $\mathbf{k}$ Scatterplot showing relationship between gain of methylation in Dnmt3a $4 / D 329 A$ and initial levels of H3K27me3 enrichment. I Scatterplot showing relationship between change in gene expression and change in respective gene promoter H3K4me3 enrichment between $\mathrm{Dnmt3} \mathrm{a}^{+/+}$and Dnmt3a $\Delta / \mathrm{D} 329 \mathrm{~A}$ adult hypothalamus. H3K4me3 enrichment was quantitated over gene promoters. In $\mathbf{e}, \mathbf{g}$, $\mathbf{h}$, $\mathbf{i}$ Low level: bottom quintile (0-20\%) of enrichment;high level: top quintile (80-100\%) of enrichment. In e-i, boxplots show median value and 25-75th percentiles, whiskers show lowest and highest observation, excluding outliers marked by individual points. Raw data are provided in Source Data. In $\mathbf{g}$-i, histone mark enrichments were quantitated over 100-CpG DMR tiles

of these hypomethylated, H3K27me3-marked domains involves a complex and still poorly understood interplay between PRC2, DNMT3A, and DNA demethylases (TET proteins) (Fig. 9a). There has been considerable focus on what defines the boundaries of these domains, which is thought to rely on a dynamic equilibrium between DNMT3A and TET proteins ${ }^{60,61,70,71}$. Knockouts of either protein strongly affect domain boundaries, whilst the core regions remain protected through an unknown mechanism. The protection of these domains is exemplified by a $D n m t 3 b$ overexpression mouse model that shows aberrant gain of methylation genome-wide and a marginal increase $(<10 \%)$ across H3K27me3-marked domains ${ }^{75}$. While DNMT3s have a strong affinity towards $\mathrm{H} 3 \mathrm{~K} 36 \mathrm{me} / 3$, there may be a weak affinity towards $\mathrm{H} 3 \mathrm{~K} 27 \mathrm{me} 3$, such that the abundance of different DNMT3s and their interacting proteins could modulate chromatin localisation depending on the cellular context. A direct association of DNMTs with $\mathrm{H} 3 \mathrm{~K} 27 \mathrm{me} 3$ regions in some contexts is supported by the observation that DNA methylation is dependent on the deposition of $\mathrm{H} 3 \mathrm{~K} 27 \mathrm{me} 3$ at a subset of CGIs in extraembryonic ectoderm ${ }^{76}$ and cancer ${ }^{77}$. At this time, it is unclear how the D329A mutation may have altered the properties of DNMT3A to enhance its interaction with H3K27me3 and/or with members of the PRC2, allowing it not only to target the boundaries but the protected core of bivalent domains as well as (Fig. 9b). One possibility is that $\mathrm{H} 3 \mathrm{~K} 36 \mathrm{me} 2 / 3$ acts as a sink for wild-type DNMT3A, and loss of affinity for this interaction would permit mutant protein to localise to bivalent chromatin exclusively, tipping the balance between DNMT3A and TETs at those sites.

As both DNA methylation and $\mathrm{H} 3 \mathrm{~K} 27 \mathrm{me} 3$ are repressive modifications, it is rather surprising to see de-repression of strongly silenced genes in $D n m t 3 a^{\Delta / D 329 A}$ hypothalamus, when methylation is acquired and $\mathrm{H} 3 \mathrm{~K} 27 \mathrm{me} 3$ is maintained to some extent. In a similar observation, Hox genes were activated when DNA methylation was targeted to gene bodies within these regions in tumours ${ }^{78}$. Yet, in contrast, we find that DNA methylation is acquired across the whole domain, not just gene bodies. Together, these findings suggest that aberrant targeting of DNA methylation to the core of bivalent domains could lead to displacement of PRC2, disrupting the repressive chromatin conformation. This is supported by the observation that DNA methylation generally, albeit not always, inhibits PRC2 binding and activity in vitro ${ }^{65,79}$ and can block the deposition of $\mathrm{H} 3 \mathrm{~K} 27 \mathrm{me}^{66}$. Furthermore, aberrant methylation gain over PRC2 domains leads to loss of H3K27me3 when DNMT3B is overexpressed ${ }^{75}$. The $\mathrm{H} 3 \mathrm{~K} 27 \mathrm{me} 3$ that remains at the hypermethylated DMRs we observed could be explained by lower histone turnover rates in non-replicative neurons or could be maintained by PRC2 variants that show affinity for methyl-CpG ${ }^{79}$.

Despite Dnmt3a being expressed throughout prenatal and postnatal development, we only observed hypermethylation of DNA in the presence of the Dnmt $3 a^{D 329 A}$ allele postnatally. In prenatal development, the predominant transcript isoform is Dnmt3a2, while in postnatal tissues it is Dnmt3a1 19,70 . The presence of the mutation specifically in the longer protein derived from isoform 1 may enable the aberrant targeting to bivalent chromatin, especially since DNMT3A1 has been found to associate with $\mathrm{H} 3 \mathrm{~K} 27 \mathrm{me} 3$-marked shores of bivalent CGIs ${ }^{70,71}$. Alternatively, Dnmt3b is very highly expressed throughout early prenatal development and is responsible for the majority of de novo methylation ${ }^{20}$. Therefore, in this context, the aberrant localisation of the mutant DNMT3A may be rescued by heterodimerisation with DNMT3B ${ }^{80,81}$.

Recently, point mutations in the PWWP domain of human DNMT3A have been described in a specific class of tumourhereditary paraganglioma ${ }^{82}$ and in microcephalic dwarfism ${ }^{27}$, including a substitution in the amino acid residue orthologous to murine D329. In both cases, the mutations are associated with gain of methylation of $\mathrm{H} 3 \mathrm{~K} 27$ me3-marked domains containing developmental regulatory genes, similar to the findings in our study. Moreover, a W326R mutation induced in mice exhibits a similar dominant growth effect and hypermethylation, although the molecular mechanisms remain to be fully explored ${ }^{27}$. Importantly, in our study, the detailed analysis of the ectopic DNA methylation in relation to chromatin data suggests a positive recruitment of the DNMT3A-PWWP mutant protein to $\mathrm{H} 3 \mathrm{~K} 27 \mathrm{me} 3$-marked domains, rather than non-selective relocalisation from $\mathrm{H} 3 \mathrm{~K} 36 \mathrm{me} 2 / 3$ sites favoured by Heyn et al. ${ }^{27}$, as we do not observe gains of DNA methylation across other genome features. Nevertheless, future studies will be invaluable in elucidating the biochemical properties of the mutated DNMT3A PWWP domain.

In summary, we provide a characterisation of the growth phenotype and underlying molecular changes of the Dnmt3a $a^{D 29 A}$ mutant mouse model. We demonstrate that the mutant DNMT3A exhibits highly specific, altered targeting to H3K27me3-marked chromatin. The aberrant gain in DNA methylation across these domains is only observed in postnatal development, suggesting that the DNMT3A1 isoform may be uniquely affected or that the absence of DNMT3B in adult tissues unmasks this effect. This study provides further insights into the 
a

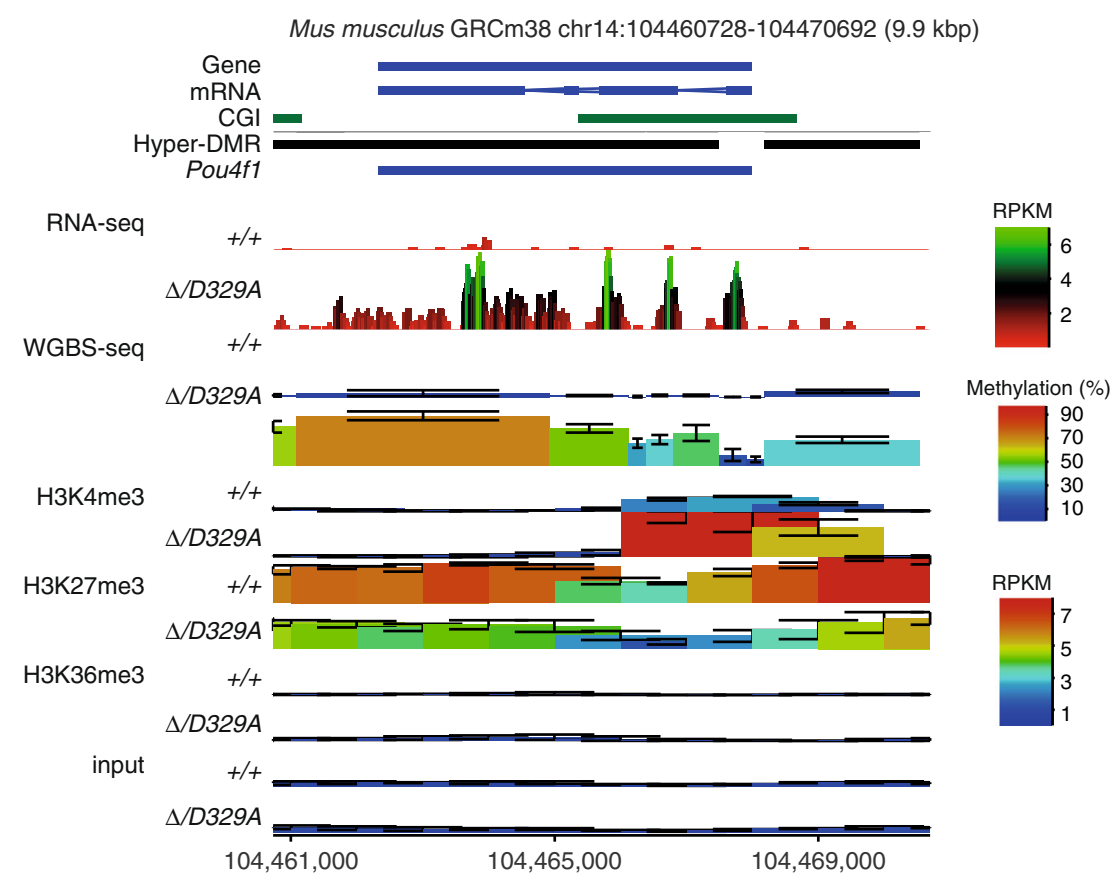

b

Mus musculus GRCm38 chr4:115043284-115080249 (36.9 kbp)
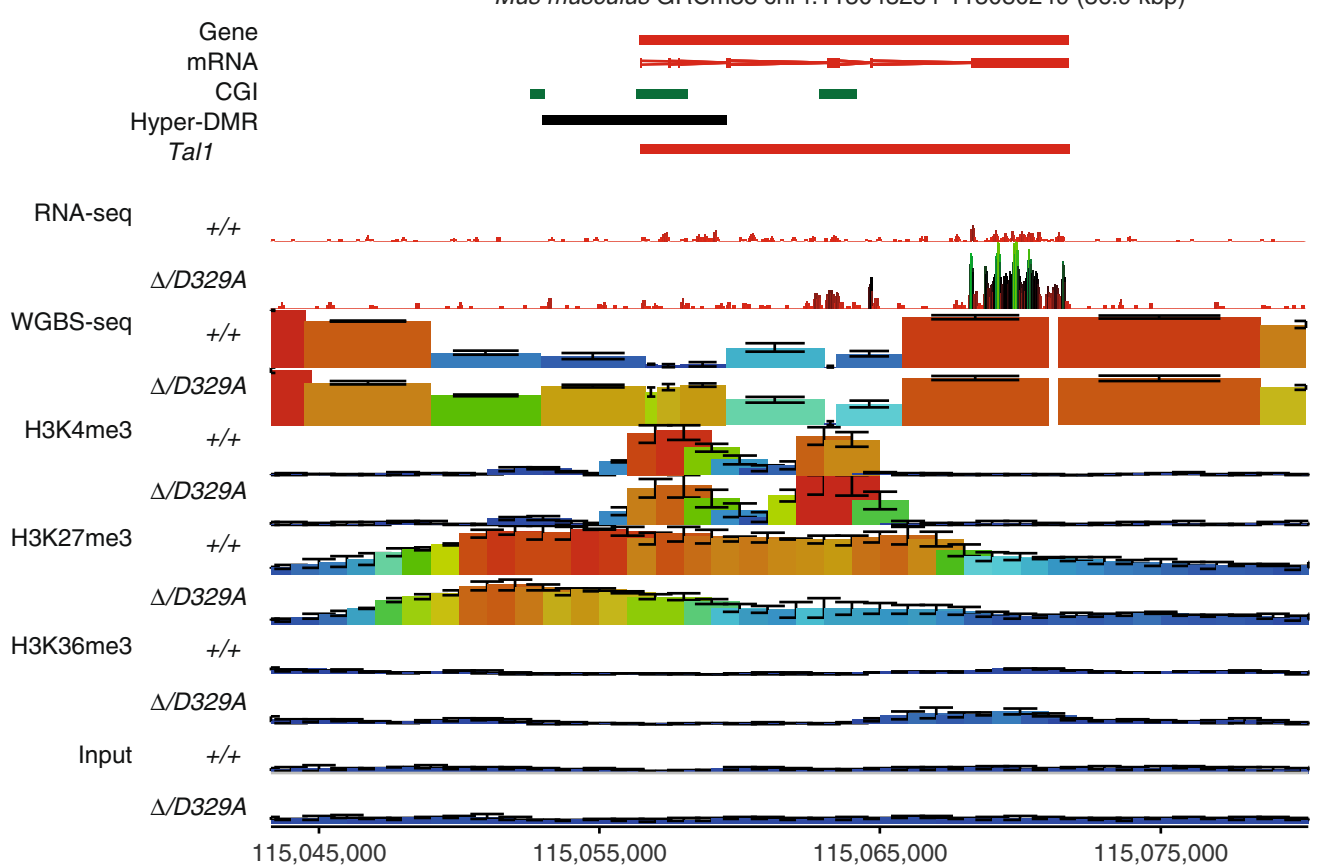

Fig. 7 Genomic landscape of de-repressed genes in Dnmt3a $4 / D 329 A$ hypothalamus. a, b Representative genome browser views of gene expression, DNA methylation, and distribution of H3K4me3, H3K27me3, and H3K36me3 marks (determined by ChIP-seq) in adult (14-week) male hypothalamus of Dnmt3a $a^{+/+}$and Dnmt3a $1 / D 329 A$ genotypes. H3K4me3 and H3K36me3 are enriched over actively transcribed CGI promoters and gene bodies, respectively. H3K27me3 shows a broad enrichment over transcriptionally silent genes and loss of enrichment upon methylation gain. Genes a Pou4f1 and $\mathbf{b}$ Tal1 are upregulated and hypermethylated in Dnmt3a $a^{\Delta} 329 \mathrm{~A}$. CGI: CpG island, DMR: hypermethylated region. Each colour-coded block represents: a 50 bp window with $10 \mathrm{bp}$ step size in the gene expression dataset; a 100-CpG window in the methylation dataset; and $2 \mathrm{~kb}$ window with $1 \mathrm{~kb}$ step size in the histone enrichment datasets. For gene and mRNA tracks, the colour indicates direction, where red is a forward strand and blue is a reverse strand. Error bars indicate standard deviation. $n(+/+)=5, n(\Delta / D 392 A)=4$ for RNA-seq datasets; $n=3$ for each genotype in WGBS-seq and ChIP-seq datasets

regulation of bivalent chromatin domains and the role of the DNMT3A PWWP domain. This model will be valuable for future investigations into the role of DNA methylation abnormalities in cancer and the growth regulatory function of DNMT3A.

\section{Methods}

Animal experimental procedures and sample collection. All animal experimental procedures were approved by the Animal Welfare and Ethical Review body at the Babraham Institute, and were conducted under authority of the UK Home Office issued licences in accordance with the Animal (Scientific Procedures) Act 1986 . The Dnmt $3 a^{D 329 A}$ mutant strain was generated by GenOway 
a

Mus musculus GRCm38 chr2:74578142-74879476 (301.3 kbp)

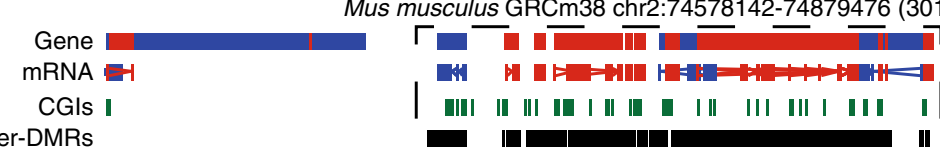

Hyper-DMRs

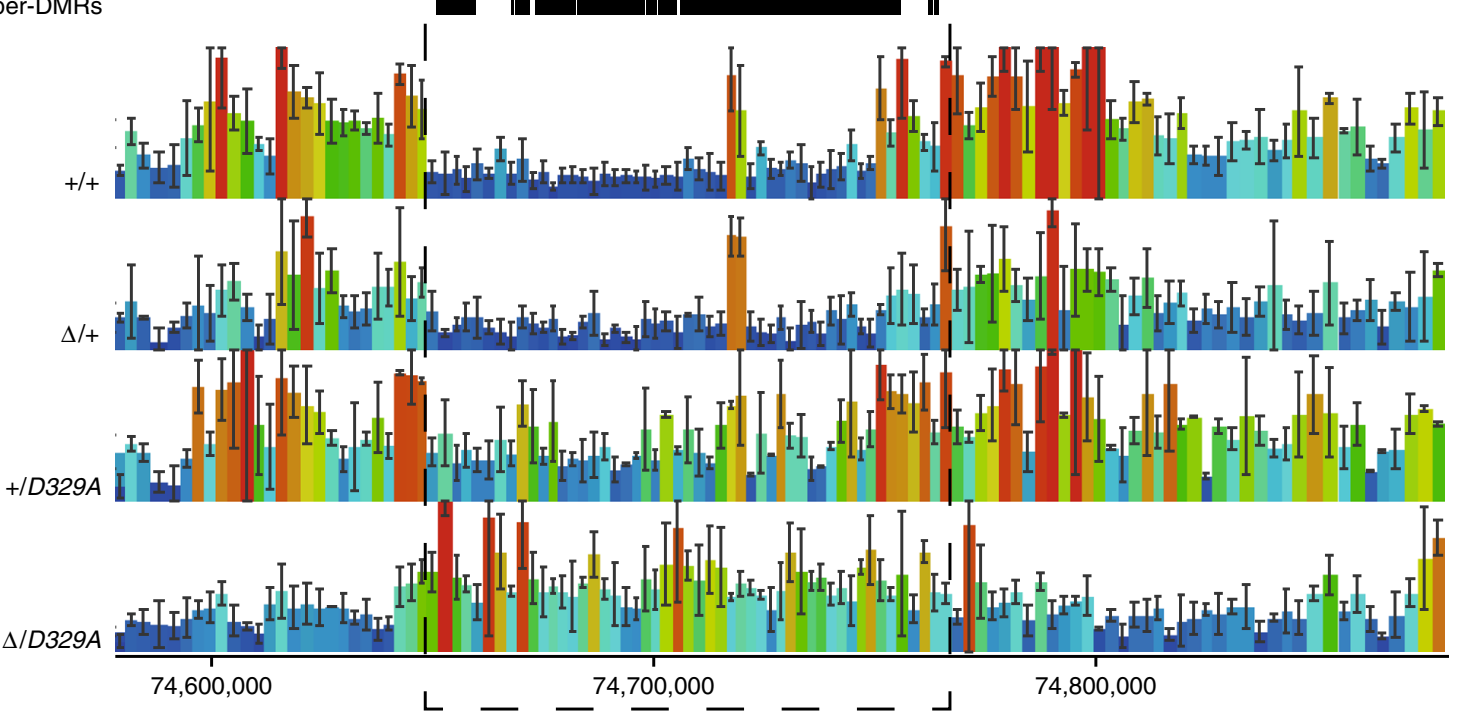

b

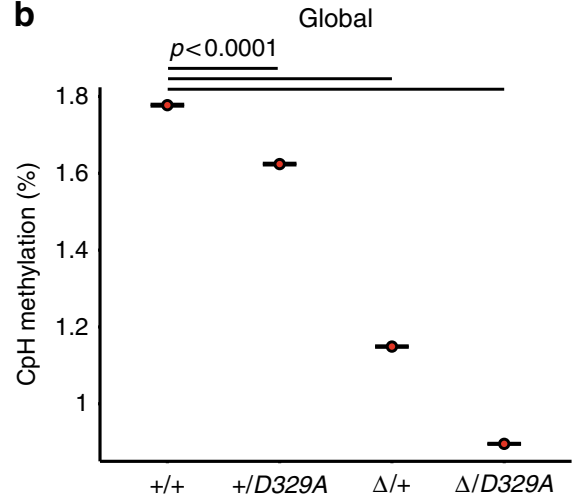

C

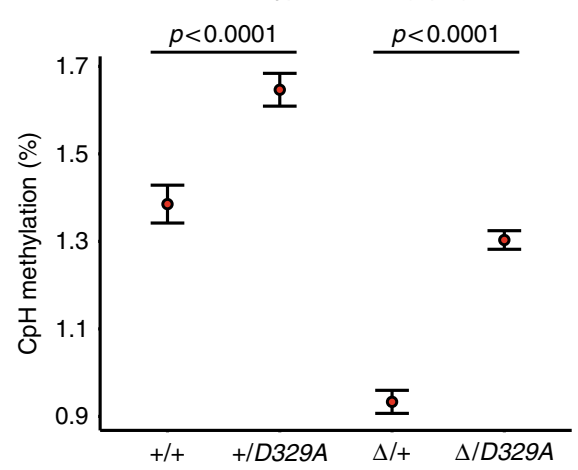

d

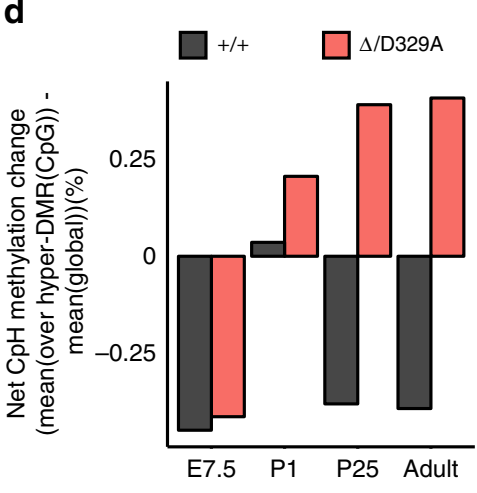

Fig. $8 \mathrm{CpH}$ methylation in hypothalamus of $D n m t 3 a^{+/+}$and $D n m t 3 a^{\Delta / D 329 A}$ mice. a Representative genome browser view of $\mathrm{CpH}$ methylation in adult (14week) male hypothalamus. Methylation levels appear to be dependent on the number of alleles present, with a decrease observed in Dnmt3a $\Delta /+$ compared to $D n m t 3 a^{+/+}$. Notably, there is an increase of methylation over hyper-DMR regions (highlighted by the box) in the presence of Dnmt3aD329A. Each bar represents a $1000-\mathrm{CpH}$ tile. For gene and mRNA tracks, the colour indicates direction, where red is a forward strand and blue is a reverse strand. CGI: $\mathrm{CpG}$ island, hyper-DMR: hypermethylated region. $n(+/+, \Delta /+, \Delta / D 329 A)=3, n(+/ D 329 A)=2$. Error bars indicate standard deviation. $\mathbf{b} \mathrm{Global}$ mean $\mathrm{CpH}$ methylation values across different genotypes. c Mean $\mathrm{CpH}$ methylation values over DMRs with $\mathrm{CpG}$ hypermethylation. $\mathbf{b}, \mathbf{c} 1000-\mathrm{CpH}$ tile quantitation for chromosomes 2 and 11 was used as representative. Error bars indicate standard error of the mean. Pairwise comparisons were done using a two-tailed $t$-test. Raw data are provided in Source Data. $\mathbf{d}$ Barplot indicating the net difference in mean $\mathrm{CpH}$ methylation between hyper-DMRs and global levels for Dnmt3at/+ and Dnmt3a ${ }^{\Delta / D 329 A}$ mice across development (E7.5 epiblast, P1, P25, and adult hypothalamus). Raw data are provided in Source Data

(France) by targeting in C57BL/6 ES cells. Briefly, a point mutation GAT to GCT resulting in aspartic acid to alanine amino acid change at the endogenous locus of Dnmt3a codon 329 was introduced (Fig. 1a and Supplementary Fig. 1).

Targeting documentation is available upon request. These mice were subsequently inter-crossed with C57BL/6Babr mice carrying a conditional Dnmt3a deletion allele Dnmt3all and oocyte-specific Zp3 promoter-driven Cre recombinase $^{12}$. In order to obtain experimental litters Dnmt3a $a^{f-/+} \mathrm{Zp} 3-\mathrm{Cr} e^{+v e}$ females, which carry oocytes with a conditionally deleted $D n m t 3 a^{\Delta /+}$ allele, were crossed with Dnmt3aD329A/+ males; yielding litters with four possible genotypes Dnmt3a $a^{+/+}, D n m t 3 a^{\Delta /+}, D n m t 3 a^{+/ D 329 A}$, and Dnmt3a $a^{\Delta / D 329 A}$ (Fig. 1b). Matings, confirmed pregnancies, litters and pup numbers were recorded, where the Dnmt $3 a^{D 329 A}$ allele was passed through the maternal or paternal line in mating with wild-type C57BL/6Babr mice. Caesarean sections were performed to rescue the pups if Dnmt3a+/D329A females failed to deliver. When possible, body weights of experimental litters were recorded at $1.4,4,6,8,10$, and 12 weeks and analysed using mixed-model ANOVA with post hoc pairwise comparisons using Bonferroni correction. Litters were sacrificed and tissues were collected at embryonic day 7.5 (E7.5), postnatal day 1 (P1), P25, and adulthood ( 14 weeks old). At E7.5 ectoplacental cone (EPC) and epiblast were collected in PBS and EZ nuclear lysis buffer (Sigma), respectively.
Genotyping. E7.5 EPCs were lysed in embryo lysis buffer (1 M Tris pH8.5, $0.5 \mathrm{M}$ EDTA pH 8, 10\% Tween, $0.2 \mathrm{mg} / \mathrm{ml}$ Proteinase K) and used for genotyping by PCR with primers for Dnmt3att/f// alleles (D3A-F $5^{\prime}$-CTGTGGCATC TCAGGGTGAT GAGCA-3', D3A-R1 5'-GCAAACAGAC CCAACATGGA ACCCT-3', and D3AR2 5'-TGAGTGGTGA GGCCCAGCTT ATCGA-3') and Dnmt3aD329A allele

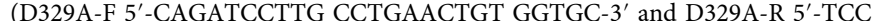
CTCTTGG TCCAGCATGT ACCCT-3'). Adult mice were genotyped by the same PCR assay using genomic DNA extracted from ear clips, or by service provider Transnetyx (US) using real-time PCR.

ELISA. Blood samples from experimental animal hearts were collected into Microvette $500 \mathrm{Z}$-Gel tubes (Sartstedt), centrifuged for $5 \mathrm{~min}$ at $10,000 \times g$ at RT. Serum collected was used in Rat/Mouse Growth Hormone ELISA kit (Millipore) and Quantikine ELISA for Mouse/Rat Igf-1 kit (R\&D systems) as per manufacturers' instructions.

RNA-sequencing library preparation. Total RNA from 14-week-old animal hypothalami was extracted using TRIzol (Invitrogen) and cleaned using RNA Clean and Concentrator (Zymo research) and Ribo-Zero rRNA removal kit 
a

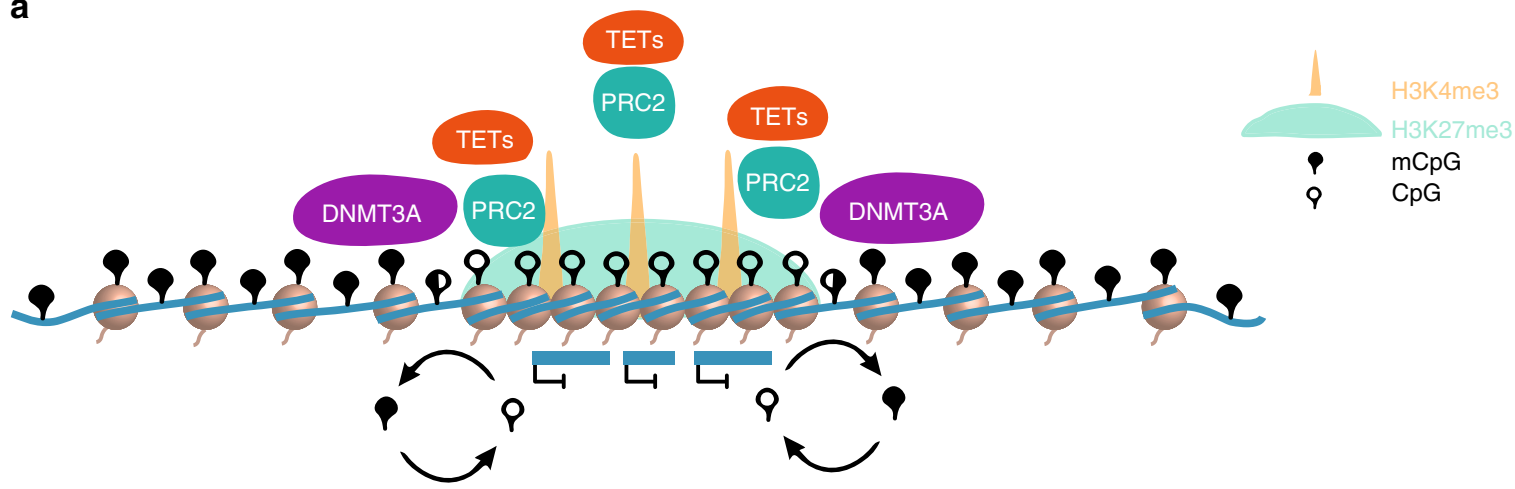

b

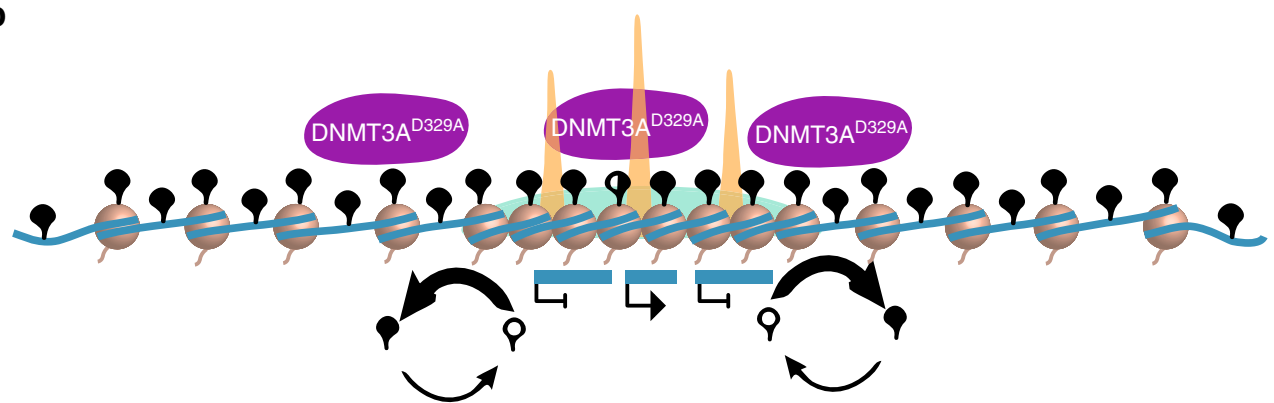

Fig. 9 Model of molecular mechanisms acting at bivalent domains. a Representation of current understanding of bivalent domain regulation. The PRC2 complex deposits H3K27me3 and compacts chromatin across the promoters and surrounding regions of transcriptionally silent, developmental genes ${ }^{62}$. TET proteins are recruited, which result in active removal of DNA methylation and protection of bivalent chromatin from de novo DNMTs ${ }^{71}$. DNMT3A is recruited to bivalent chromatin shores through an unknown mechanism, enabling active establishment of DNA methylation at the boundaries $60,70,71$. Through the competing actions of TETs and DNMT3A there is a cycle of DNA methylation turnover at the boundaries of bivalent domains. $\mathbf{b}$ Representation of bivalent chromatin dynamics in the presence of DNMT3A $329 \mathrm{~A}$. The mutant protein is able to access bivalent chromatin and establish methylation across the whole domain. The presence of DNA methylation appears to influence the local chromatin environment and results in reduction of H3K27me3, potentially due to exclusion of PRC2 from methylated DNA. As a consequence, some genes become de-repressed and show an increase in promoter H3K4me3, while others maintain their transcriptional silencing, this is likely dependent on the availability and strength of necessary transcription factors

(Illumina). Libraries were generated using NEBNext Ultra RNA library preparation kits (New England Biolabs). RNA-seq libraries were sequenced $50 \mathrm{bp}$ single-end using Illumina HiSeq2500 platform.

RNA-seq analysis. RNA-seq data was trimmed using TrimGalore $0.5 .0^{83}$, then mapped using HiSat2 2.1.0 ${ }^{84}$ to Mus musculus genome GRCm38. Processed data was analysed using Seqmonk $1.42 .0^{85}$. Reads over transcripts were merged across exons correcting for feature length, quantitated using RNA-Seq quantitation pipeline, and $\log _{2}$-transformed. Differentially expressed genes were called using DESeq with significance threshold of $p<0.01$ after Benjamini-Hochberg multiple testing correction. Mitochondrial genes were excluded. For genome browser views of gene expression selected regions were split into $500 \mathrm{bp}$ bins every $50 \mathrm{bp}$ and quantitated as reads per million transcripts, unless specified otherwise. David $6.7^{86}$ Functional Annotation Tool was used for gene ontology analysis, with categories GOTERM_BP_FAT, GOTERM_CC_FAT, GOTERM_MF_FAT, using Benjaminicorrected $p<0.01$ significance cut-off.

PBAT sequencing library preparation. Post-bisulphite adaptor tagging (PBAT) was used to generate genome-wide DNA methylation profiles. Approximately $50 \mathrm{ng}$ of gDNA or $10 \%$ of MNase-digested E7.5 Epiblast DNA, purified using solid phase reversible immobilisation (SPRI) beads, was used as an input material. Briefly, Imprint DNA modification kit (Sigma) one-step procedure was used to bisulphite-convert the DNA. First strand synthesis was done using Klenow exo-tagged (New England Biolabs) and biotin-tagged Illumina $9 \mathrm{bp}$ random sequence adaptor, followed by Exo-I treatment (New England Biolabs) and SPRI purification. Biotinylated DNA was captured using Dynabeads Strepatavidin M-280 beads (ThermoFisher Scientific) and second strand synthesis was carried out using reverse Illumina $9 \mathrm{bp}$ random sequence adaptor, followed by library amplification using Phusion HF (Thermo Scientific) or KAPA HiFi (Kapa Biosystems) polymerase for 10 cycles. Libraries were quantitated and quality control checked using Agilent High Sensitivity DNA kit and Kapa Illumina library quantitation kit, as per manufacturer's instructions. Libraries were sequenced using $100 \mathrm{bp}$ single-end for P1, P25, and adult tissues; and $150 \mathrm{bp}$ for E7.5 epiblast sequencing mode on the Illumina NextSeq500 platform at the Babraham Institute Sequencing Facility.
Methylation data analysis. Methylation data was trimmed using TrimGalore 0.5. $0^{83}$, deduplicated, mapped to Mus musculus genome GRCm38, and methylation calls were extracted using Bismark 0.19.187. Processed data was analysed using Seqmonk $1.42 .0^{85}$. Pituitary datasets were down-sampled to the least covered dataset using SeqMonk to avoid differential coverage artefacts. Probes were defined as tiles of CpG positions and quantified using Bisulphite quantitation pipeline. Tiles in adult hypothalamus were 100-CpGs where at least 10 are covered. In pituitary, liver, E7.5 epiblast and when E7.5 epiblast data was used in analysis, tiles were 300-CpG where at least 10 are covered. Mitochondrial genes were excluded from analyses, $\mathrm{X}$ and $\mathrm{Y}$ genes were excluded only from E7.5 epiblast analyses. DMRs were called using Seqmonk edgeR (for/rev) statistics filter with absolute methylation difference cut-off of $20 \%$, using multiple-testing corrected $p$-value of $\leq 0.01$. Genomic locations were defined as listed in the brackets using feature annotations available in SeqMonk: promoter $(-1500$ to +500 bp around transcription start site), gene body $(+500$ bp to the end of the mRNA/gene), intergenic (promoters and gene bodies excluded). CGI features were called based on previously published CGI coordinate data ${ }^{88}$. For promoter analysis, $100 \mathrm{CpG}$ windows were fused together if distance between them were $<1 \mathrm{~kb}$. For intergenic regions, adjacent probes were fused together. Random probes were selected from all probes, excluding those in the DMR list, and utilised as representative of the genome. Previously described DNA methylation 'canyon' coordinates were used in the analysis ${ }^{60}$. Only those showing methylation under $10 \%$ and covered by at least 5 CpGs in hypothalamus were used in analysis. Non-CpG methylation was analysed using $1000 \mathrm{CpH}$ tiles where at least $30 \mathrm{CpHs}$ were covered.

Ultra-low input native ChIP-seq library preparation. Ultra-low input native chromatin immunoprecipitation sequencing (ChIP-seq) libraries were generated using $2.5 \%$ of a whole hypothalamus as input for each immunoprecipitation, following previously described protocol ${ }^{89}$, keeping $10 \%$ of that for input. Briefly, cells were permeabilised using triton X-100/deoxycholate, chromatin was then digested using micrococcal nuclease (New England Biolabs) and precleared using Dynabeads Protein A/G beads (ThermoFisher Scientific). Antibodies for H3K4me3 (250 ng per reaction, Diagenode C15410003), H3K27me3 (125 ng per reaction, Millipore 07-449), or H3K36me3 (250 ng per reaction, Diagenode C15410192) were pre-bound to Protein A/G beads and incubated with chromatin for immunoprecipitation. Bound DNA was purified by SPRI purification. MicroPlex Library 
Preparation kit v2 (Diagenode) was used as per manufacturer's instructions to generate libraries of inputs and immunoprecipitated samples. Libraries were quantitated and quality control checked using Agilent High Sensitivity DNA kit and Kapa Illumina library quantitation kit, as per manufacturer's instructions. Libraries were sequenced using $75 \mathrm{bp}$ single-end sequencing on the Illumina NextSeq500 platform at the Babraham Institute Sequencing Facility.

ChIP data analysis. ChIP-seq data was trimmed using Trim Galore $0.5 .0^{83}$ and mapped against Mus Musculus GRCm38 genome with Bowtie 1.2.290. H3K27me3 enrichment was quantitated using $2 \mathrm{~kb}$ tiles with a $1 \mathrm{~kb}$ step, correcting for total count based on the largest library, unless stated otherwise. H3K4me3 and H3K27me3 peaks were called using MACS peak caller within SeqMonk 1.42.0, using a significant threshold of $p<0.00001$, a sonication fragment size of $1 \mathrm{~kb}$, and input controls as a reference. When assessment of histone marks was done in relation to DNA methylation, DNA methylation tiles were used to quantitate the enrichment.

Statistical analyses. All measurements taken were biological and not technical replicates, replicates represent individual animals. Basic statistical tests used are described in figure legends where applicable

Reporting summary. Further information on experimental design is available in the Nature Research Reporting Summary linked to this article.

\section{Data availability}

Sequence data that support the findings of this study have been deposited in Gene Expression Omnibus with the primary accession code GSE117728. All data generated and analysed in this study are included in the published article and its supplementary files and are available from the corresponding author upon reasonable request. Source data for Figs. 1c, 2a, 3a, 4a, b, 6e-i, 8b, c and Supplementary Figs. 3, 4a, 4c, d, 5a, b, 11a, $11 \mathrm{f}-\mathrm{i}$ are provided. Source data for Figs. $2 \mathrm{c}, \mathrm{d}$ and $5 \mathrm{~b}$ are provided in Supplementary Data. A Life Science and ChIP-Seq Reporting Summary for this article is available.

Received: 10 December 2018 Accepted: 26 March 2019

Published online: 23 April 2019

\section{References}

1. Smith, Z. D. \& Meissner, A. DNA methylation: roles in mammalian development. Nat. Rev. Genet. 14, 204-220 (2013).

2. Yin, Y. et al. Impact of cytosine methylation on DNA binding specificities of human transcription factors. Science 356, eaaj2239 (2017).

3. Borgel, J. et al. Targets and dynamics of promoter DNA methylation during early mouse development. Nat. Genet. 42, 1093-1100 (2010).

4. Jones, P. A. Functions of DNA methylation: islands, start sites, gene bodies and beyond. Nat. Rev. Genet. 13, 484-492 (2012).

5. Smith, Z. D. et al. A unique regulatory phase of DNA methylation in the early mammalian embryo. Nature 484, 339-344 (2012).

6. Ziller, M. J. et al. Charting a dynamic DNA methylation landscape of the human genome. Nature 500, 477-481 (2013).

7. Ehrlich, M. \& Wang, R. Y. 5-Methylcytosine in eukaryotic DNA. Science 212, 1350-1357 (1981).

8. Okano, M., Xie, S. \& Li, E. Cloning and characterization of a family of novel mammalian DNA (cytosine-5) methyltransferases. Nat. Genet. 19, 219-220 (1998).

9. Barau, J. et al. The DNA methyltransferase DNMT3C protects male germ cells from transposon activity. Science 354, 909-912 (2016).

10. Jain, D. et al. rahu is a mutant allele of Dnmt3c, encoding a DNA methyltransferase homolog required for meiosis and transposon repression in the mouse male germline. PLoS. Genet. 13, el006964 (2017).

11. Li, E., Bestor, T. H. \& Jaenisch, R. Targeted mutation of the DNA methyltransferase gene results in embryonic lethality. Cell 69, 915-926 (1992).

12. Chuang, L. S.-H. et al. Human DNA-(cytosine-5) methyltransferase-PCNA complex as a target for p21WAF1. Science 277, 1996-2000 (1997).

13. Bostick, M. et al. UHRF1 plays a role in maintaining DNA methylation in mammalian cells. Science 317, 1760-1764 (2007)

14. Sharif, J. et al. The SRA protein Np95 mediates epigenetic inheritance by recruiting Dnmtl to methylated DNA. Nature 450, 908-912 (2007).

15. Bourc'his, D., Xu, G.-L., Lin, C.-S., Bollman, B. \& Bestor, T. H. Dnmt3L and the establishment of maternal genomic imprints. Science 294, 2536-2539 (2001).

16. Hata, K., Okano, M., Lei, H. \& Li, E. Dnmt3L cooperates with the Dnmt3 family of de novo DNA methyltransferases to establish maternal imprints in mice. Development 129, 1983-1993 (2002).

17. Bourc'his, D. \& Bestor, T. H. Meiotic catastrophe and retrotransposon reactivation in male germ cells lacking Dnmt3L. Nature 431, 96-99 (2004).
18. Okano, M., Bell, D. W., Haber, D. A. \& Li, E. DNA methyltransferases Dnmt3a and Dnmt3b are essential for de novo methylation and mammalian development. Cell 99, 247-257 (1999).

19. Chen, T., Ueda, Y., Xie, S. \& Li, E. A novel Dnmt3a isoform produced from an alternative promoter localizes to euchromatin and its expression correlates with activede novo methylation. J. Biol. Chem. 277, 38746-38754 (2002).

20. Auclair, G., Guibert, S., Bender, A. \& Weber, M. Ontogeny of CpG island methylation and specificity of DNMT3 methyltransferases during embryonic development in the mouse. Genome Biol. 15, 545 (2014).

21. Kaneda, M. et al. Essential role for de novo DNA methyltransferase Dnmt3a in paternal and maternal imprinting. Nature 429, 900-903 (2004).

22. Kaneda, M. et al. Genetic evidence for Dnmt3a-dependent imprinting during oocyte growth obtained by conditional knockout with $\mathrm{Zp3}$-Cre and complete exclusion of Dnmt3b by chimera formation. Genes Cells 15, 169-179 (2010).

23. Feng, J., Chang, H., Li, E. \& Fan, G. Dynamic expression of de novo DNA methyltransferases Dnmt3a and Dnmt3b in the central nervous system. J. Neurosci. Res. 79, 734-746 (2005).

24. Tatton-Brown, K. et al. Mutations in the DNA methyltransferase gene DNMT3A cause an overgrowth syndrome with intellectual disability. Nat. Genet. 46, 385-388 (2014)

25. Yang, L., Rau, R. \& Goodell, M. A. DNMT3A in haematological malignancies. Nat. Rev. Cancer 15, 152-165 (2015).

26. Tatton-Brown, K. et al. Mutations in epigenetic regulation genes are a major cause of overgrowth with intellectual disability. Am. J. Hum. Genet. 100, 725-736 (2017).

27. Heyn, P. et al. Gain-of-function DNMT3A mutations cause microcephalic dwarfism and hypermethylation of Polycomb-regulated regions. Nat. Genet. 51, 96 (2019).

28. Hansen, R. S. et al. The DNMT3B DNA methyltransferase gene is mutated in the ICF immunodeficiency syndrome. Proc. Natl Acad. Sci. USA 96, 14412-14417 (1999)

29. Dodge, J. E., Ramsahoye, B. H., Wo, Z. G., Okano, M. \& Li, E. De novo methylation of MMLV provirus in embryonic stem cells: CpG versus nonCpG methylation. Gene 289, 41-48 (2002).

30. Ramsahoye, B. H. et al. Non-CpG methylation is prevalent in embryonic stem cells and may be mediated by DNA methyltransferase 3a. Proc. Natl Acad. Sci. USA 97, 5237-5242 (2000).

31. Wienholz, B. L. et al. DNMT3L modulates significant and distinct flanking sequence preference for DNA methylation by DNMT3A and DNMT3B in vivo. PLoS Genet. 6, e1001106 (2010)

32. Jeltsch, A., Broche, J. \& Bashtrykov, P. Molecular processes connecting DNA methylation patterns with DNA methyltransferases and histone modifications in mammalian genomes. Genes 9, 566 (2018).

33. Argentaro, A. et al. Structural consequences of disease-causing mutations in the ATRX-DNMT3-DNMT3L (ADD) domain of the chromatin-associated protein ATRX. PNAS 104, 11939-11944 (2007).

34. Otani, J. et al. Structural basis for recognition of H3K4 methylation status by the DNA methyltransferase 3A ATRX-DNMT3-DNMT3L domain. EMBO Rep. 10, 1235-1241 (2009).

35. Ooi, S. K. T. et al. DNMT3L connects unmethylated lysine 4 of histone $\mathrm{H} 3$ to de novo methylation of DNA. Nature 448, 714-717 (2007).

36. Zhang, Y. et al. Chromatin methylation activity of Dnmt3a and Dnmt3a/3L is guided by interaction of the ADD domain with the histone $\mathrm{H} 3$ tail. Nucleic Acids Res. 38, 4246-4253 (2010).

37. Guo, X. et al. Structural insight into autoinhibition and histone H3-induced activation of DNMT3A. Nature 517, 640-644 (2015).

38. Li, B.-Z. et al. Histone tails regulate DNA methylation by allosterically activating de novo methyltransferase. Cell Res. 21, 1172-1181 (2011).

39. Qin, S. \& Min, J. Structure and function of the nucleosome-binding PWWP domain. Trends Biochem. Sci. 39, 536-547 (2014).

40. Chen, T., Tsujimoto, N. \& Li, E. The PWWP domain of Dnmt3a and Dnmt3b Is required for directing DNA methylation to the major satellite repeats at pericentric heterochromatin. Mol. Cell. Biol. 24, 9048-9058 (2004).

41. Ge, Y.-Z. et al. Chromatin targeting of de Novo DNA methyltransferases by the PWWP domain. J. Biol. Chem. 279, 25447-25454 (2004).

42. Shirohzu, H. et al. Three novel DNMT3B mutations in Japanese patients with ICF syndrome. Am. J. Med. Genet. 112, 31-37 (2002).

43. Rondelet, G., Dal Maso, T., Willems, L. \& Wouters, J. Structural basis for recognition of histone H3K36me3 nucleosome by human de novo DNA methyltransferases 3A and 3B. J. Struct. Biol. 194, 357-367 (2016).

44. Dhayalan, A. et al. The Dnmt3a PWWP domain reads histone 3 lysine 36 trimethylation and guides DNA methylation. J. Biol. Chem. 285, 26114-26120 (2010).

45. Bock, I. et al. Application of Celluspots peptide arrays for the analysis of the binding specificity of epigenetic reading domains to modified histone tails. BMC Biochem. 12, 48 (2011).

46. Kungulovski, G. et al. Application of histone modification-specific interaction domains as an alternative to antibodies. Genome Res. 24, 1842-1853 (2014). 
47. Mauser, R., Kungulovski, G., Keup, C., Reinhardt, R. \& Jeltsch, A. Application of dual reading domains as novel reagents in chromatin biology reveals a new $\mathrm{H} 3 \mathrm{~K} 9 \mathrm{me} 3$ and $\mathrm{H} 3 \mathrm{~K} 36 \mathrm{me} / 3$ bivalent chromatin state. Epigenetics Chromatin 10, 45 (2017).

48. Baubec, T. et al. Genomic profiling of DNA methyltransferases reveals a role for DNMT3B in genic methylation. Nature 520, 243-247 (2015).

49. Morselli, M. et al. In vivo targeting of de novo DNA methylation by histone modifications in yeast and mouse. eLife 4, e06205 (2015).

50. Greenberg, M. V. C. et al. Transient transcription in the early embryo sets an epigenetic state that programs postnatal growth. Nat. Genet. 49, 110-118 (2017).

51. Li, G. et al. Major epigenetic development distinguishing neuronal and nonneuronal cells occurs postnatally in the murine hypothalamus. Hum. Mol. Genet. 23, 1579-1590 (2014).

52. Abizaid, A. \& Horvath, T. L. Brain circuits regulating energy homeostasis. Regul. Pept. 149, 3-10 (2008).

53. Leibel, R. Molecular physiology of weight regulation in mice and humans. Int. J. Obes. 32, S98-S108 (2008).

54. Mihalache, L. et al. Effects of ghrelin in energy balance and body weight homeostasis. Hormones 15, 186-196 (2016).

55. Yang, H., An, J. J., Sun, C. \& Xu, B. Regulation of energy balance via BDNF expressed in nonparaventricular hypothalamic neurons. Mol. Endocrinol. 30, 494-503 (2016).

56. Li, X. SIRT1 and energy metabolism. Acta Biochim. Biophys. Sin. 45, 51-60 (2013).

57. Lau, J. \& Herzog, H. CART in the regulation of appetite and energy homeostasis. Front. Neurosci. 8, 313 (2014).

58. Plant, T. M. 60 YEARS OF NEUROENDOCRINOLOGY: the hypothalamopituitary-gonadal axis. J. Endocrinol. 226, T41-T54 (2015).

59. Borgel, J. et al. Targets and dynamics of promoter DNA methylation during early mouse development. Nat. Genet. 42, 1093-1100 (2010).

60. Jeong, M. et al. Large conserved domains of low DNA methylation maintained by Dnmt3a. Nat. Genet. 46, 17-23 (2014).

61. Li, Y. et al. Genome-wide analyses reveal a role of Polycomb in promoting hypomethylation of DNA methylation valleys. Genome Biol. 19, 18 (2018).

62. Boyer, L. A. et al. Polycomb complexes repress developmental regulators in murine embryonic stem cells. Nature 441, 349-353 (2006).

63. Tanay, A., O’Donnell, A. H., Damelin, M. \& Bestor, T. H. Hyperconserved CpG domains underlie Polycomb-binding sites. PNAS 104, 5521-5526 (2007).

64. Lindroth, A. M. et al. Antagonism between DNA and H3K27 Methylation at the Imprinted Rasgrf1 Locus. PLoS Genet. 4, e1000145 (2008).

65. Bartke, T. et al. Nucleosome-interacting proteins regulated by DNA and histone methylation. Cell 143, 470-484 (2010).

66. Brinkman, A. B. et al. Sequential ChIP-bisulfite sequencing enables direct genome-scale investigation of chromatin and DNA methylation cross-talk. Genome Res. 22, 1128-1138 (2012).

67. Xie, W. et al. Base-resolution analyses of sequence and parent-of-origin dependent DNA methylation in the mouse genome. Cell 148, 816-831 (2012).

68. Varley, K. E. et al. Dynamic DNA methylation across diverse human cell lines and tissues. Genome Res. 23, 555-567 (2013).

69. Lister, R. et al. Global epigenomic reconfiguration during mammalian brain development. Science 341, 1237905 (2013).

70. Manzo, M. et al. Isoform-specific localization of DNMT3A regulates DNA methylation fidelity at bivalent CpG islands. EMBO J. 36, 3421-3434 (2017).

71. Gu, T. et al. DNMT3A and TET1 cooperate to regulate promoter epigenetic landscapes in mouse embryonic stem cells. Genome Biol. 19, 88 (2018).

72. Bogdanović, O. et al. Temporal uncoupling of the DNA methylome and transcriptional repression during embryogenesis. Genome Res. 21, 1313-1327 (2011).

73. Murphy, P. J. et al. Single-molecule analysis of combinatorial epigenomic states in normal and tumor cells. PNAS 110, 7772-7777 (2013).

74. Statham, A. L. et al. Bisulfite sequencing of chromatin immunoprecipitated DNA (BisChIP-seq) directly informs methylation status of histone-modified DNA. Genome Res. 22, 1120-1127 (2012).

75. Zhang, Y. et al. Targets and genomic constraints of ectopic Dnmt3b expression. eLife 7, e40757 (2018).

76. Smith, Z. D. et al. Epigenetic restriction of extraembryonic lineages mirrors the somatic transition to cancer. Nature 549, 543-547 (2017)

77. Schlesinger, Y. et al. Polycomb-mediated methylation on Lys27 of histone H3 pre-marks genes for de novo methylation in cancer. Nat. Genet. 39, 232-236 (2007).

78. Su, J. et al. Homeobox oncogene activation by pan-cancer DNA hypermethylation. Genome. Biol. 19, 108 (2018).

79. Wang, X. et al. Molecular analysis of PRC2 recruitment to DNA in chromatin and its inhibition by RNA. Nat. Struct. Mol. Biol. 24, 1028-1038 (2017).

80. Li, J.-Y. et al. Synergistic function of DNA methyltransferases Dnmt3a and Dnmt3b in the methylation of Oct4 and Nanog. Mol. Cell. Biol. 27, 8748-8759 (2007).
81. Emperle, M., Rajavelu, A., Reinhardt, R., Jurkowska, R. Z. \& Jeltsch, A. Cooperative DNA binding and protein/DNA fiber formation increases the activity of the Dnmt3a DNA methyltransferase. J. Biol. Chem. 289, 29602-29613 (2014)

82. Remacha, L. et al. Gain-of-function mutations in DNMT3A in patients with paraganglioma. Genet. Med. 20, 1644-1651 (2018).

83. Babraham Bioinformatics-Trim Galore! http://www.bioinformatics. babraham.ac.uk/projects/trim_galore/. Accessed 10 Mar 2019.

84. Kim, D., Langmead, B. \& Salzberg, S. L. HISAT: a fast spliced aligner with low memory requirements. Nat Meth 12, 357-360 (2015).

85. Babraham Bioinformatics. SeqMonk Mapped Sequence Analysis Tool. http://www bioinformatics.babraham.ac.uk/projects/seqmonk/. Accessed 20 June 2017.

86. DAVID Functional Annotation Bioinformatics Microarray Analysis. https:// david-d.ncifcrf.gov/. Accessed 10 Mar 2019.

87. Krueger, F. \& Andrews, S. R. Bismark: a flexible aligner and methylation caller for Bisulfite-Seq applications. Bioinformatics 27, 1571-1572 (2011).

88. Illingworth, R. S. et al. Orphan CpG islands identify numerous conserved promoters in the mammalian genome. PLoS Genet. 6, e1001134 (2010).

89. Hanna, C. W. et al. MLL2 conveys transcription-independent H3K4 trimethylation in oocytes. Nat. Struct. Mol. Biol. 25, 73 (2018)

90. Langmead, B., Trapnell, C., Pop, M. \& Salzberg, S. L. Ultrafast and memory efficient alignment of short DNA sequences to the human genome. Genome Biol. 10, R25 (2009).

\section{Acknowledgements}

We would like to thank Wendy Dean, Jessica Elmer and Salah Azzi for assistance with tissue collections; Simon Andrews, Felix Krueger, and Anne Segonds-Pichon from Babraham Bioinformatics for contributions to sequencing QC and mapping, and assistance with bioinformatics and statistical analysis; Kristina Tabbada and Clare Munare of the Next Generation Sequencing Facility at the Babraham Institute; members of the Babraham Institute Biological Support Utility for dedicated mouse husbandry; and Lindsay Hamilton for veterinary support. We are also grateful to Andrew Jackson and colleagues for discussion of unpublished data. Work in G.K.'s lab is supported by grants from the UK Medical Research Council (MR/K011332/1, MR/S000437/1) and Biotechnology and Biological Sciences Research Council (BBS/E/B/000C0423); C.W.H. is supported by a University of Cambridge Centre for Trophoblast Research Next Generation Fellowship; G.S. by an MRC Doctoral Training Programme studentship; and K.R. S.-M. was supported by a scholarship from the Cambridge Overseas Trusts.

\section{Author contributions}

G.S., C.W.H., K.R.S.-M., and G.K. contributed to study design; G.S., C.W.H., K.R.S.-M., and E.I. performed experiments; G.S. and C.W.H. analysed data and generated figures; G.S., C.W.H., K.R.S.-M., and G.K. contributed to data interpretation; G.S. wrote the manuscript with input from C.W.H. and G.K.; G.K. supported the project development.

\section{Additional information}

Supplementary Information accompanies this paper at https://doi.org/10.1038/s41467019-09713-w.

Competing interests: The authors declare no competing interests.

Reprints and permission information is available online at http://npg.nature.com/ reprintsandpermissions/

Journal Peer Review Information: Nature Communications thanks Albert Jeltsch, and other anonymous reviewer(s) for their contribution to the peer review of this work. Peer reviewer reports are available.

Publisher's note: Springer Nature remains neutral with regard to jurisdictional claims in published maps and institutional affiliations.

Open Access This article is licensed under a Creative Commons Attribution 4.0 International License, which permits use, sharing, adaptation, distribution and reproduction in any medium or format, as long as you give appropriate credit to the original author(s) and the source, provide a link to the Creative Commons license, and indicate if changes were made. The images or other third party material in this article are included in the article's Creative Commons license, unless indicated otherwise in a credit line to the material. If material is not included in the article's Creative Commons license and your intended use is not permitted by statutory regulation or exceeds the permitted use, you will need to obtain permission directly from the copyright holder. To view a copy of this license, visit http://creativecommons.org/licenses/by/4.0/.

(c) The Author(s) 2019 